\title{
Exploring the diversity of Diplostomum (Digenea: Diplostomidae) in fishes from the River Danube using mitochondrial DNA barcodes
}

Olena Kudlai ${ }^{1,2,3^{*}}$, Mikuláš Oros ${ }^{4}$, Aneta Kostadinova $^{2}$ and Simona Georgieva²

\begin{abstract}
Background: Metacercariae of Diplostomum are important fish pathogens, but reliable data on their diversity in natural fish populations are virtually lacking. This study was conducted to explore the species diversity and hostparasite association patterns of Diplostomum spp. in a large riverine system in Europe, using molecular and morphological data.

Methods: Twenty-eight species of fish of nine families were sampled in the River Danube at Nyergesújfalu in Hungary in 2012 and Štúrovo in Slovakia in 2015. Isolates of Diplostomum spp. were characterised morphologically and molecularly. Partial sequences of the 'barcode' region of the cytochrome coxidase subunit 1 (cox1) and complete sequences of the nicotinamide adenine dinucleotide dehydrogenase subunit 3 (nad3) mitochondrial genes were amplified for 76 and 30 isolates, respectively. The partial cox 1 sequences were used for molecular identification of the isolates and an assessment of haplotype diversity and possible host-associated structuring of the most prevalent parasite species. New primers were designed for amplification of the mitochondrial nad3 gene.

Results: Only lens-infecting Diplostomum spp. were recovered in 16 fish species of five families. Barcoding of representative isolates provided molecular identification for three species/species-level genetic lineages, $D$. spathaceum, D. pseudospathaceum and 'D. mergi Lineage 2 ', and three single isolates potentially representing distinct species. Molecular data helped to elucidate partially the life-cycle of ' $D$. mergi Lineage 2 '. Many of the haplotypes of D. spathaceum (16 in total), D. pseudospathaceum (15 in total) and 'D. mergi Lineage 2' (7 in total) were shared by a number of fish hosts and there was no indication of genetic structuring associated with the second intermediate host. The most frequent Diplostomum spp. exhibited a low host-specificity, predominantly infecting a wide range of cyprinid fishes, but also species of distant fish families such as the Acipenseridae, Lotidae, Percidae and Siluridae. The nad3 gene exhibited distinctly higher levels of interspecific divergence in comparison with the cox1 gene.

(Continued on next page)
\end{abstract}

\footnotetext{
* Correspondence: olena.kudlai@gmail.com

${ }^{1}$ Water Research Group, Unit for Environmental Sciences and Management, Potchefstroom Campus, North-West University, Potchefstroom 2520, South Africa

${ }^{2}$ Institute of Parasitology, Biology Centre of the Czech Academy of Sciences, Branišovská 31, 37005 České Budějovice, Czech Republic

Full list of author information is available at the end of the article
} 
(Continued from previous page)

Conclusions: This first exploration of the species diversity and host ranges of Diplostomum spp., in natural fish populations in the River Danube, provided novel molecular, morphological and host-use data which will advance further ecological studies on the distribution and host ranges of these important fish parasites in Europe. Our results also indicate that the nad3 gene is a good candidate marker for multi-gene approaches to systematic estimates within the genus.

Keywords: Diplostomum, Diplostomidae, Metacercariae, Freshwater fishes, Barcodes, cox1, nad3, River Danube, Europe

\section{Background}

Metacercariae of the genus Diplostomum von Nordmann, 1832 (Digenea: Diplostomidae) are important fish pathogens [1-3] and represent a case study illustrating the difficulties of species identification based solely on morphological data. The recent use of molecular markers proved to be a valuable and efficient approach to species delimitation and identification, especially for the larval stages of Diplostomum spp. which lack reliable distinguishing morphological characters. Recent intensive molecular studies, following the publication of the genusspecific primers for the 'barcode' region of the cytochrome $c$ oxidase subunit 1 ( $\operatorname{cox} 1$ ) gene [4], resulted in the generation of sequence libraries for the North American [5, 6] and European species [3,7-12] of the genus. Thus providing a sound basis for molecular identification and provisional species delineation. These libraries provide a foundation that will allow identification of life-cycle stages and ensure an increased taxonomic resolution in epidemiological and ecological studies of these important fish parasites (e.g. Locke et al. [13]; Désilets et al. [14]; Pérez-del-Olmo et al. [3]) as well as for further exploration of species host and geographical ranges [6].

To date, molecular data for a total of 19 species/species-level genetic lineages of Diplostomum exist from North America including three named species, i.e. Diplostomum baeri Dubois, 1937, Diplostomum huronense (La Rue, 1927) and Diplostomum indistinctum (Guberlet, 1923), and 16 otherwise unidentified species or species-level lineages [4-6, 15]. Extensive studies carried out in Europe recently revealed a total of 12 species/species-level genetic lineages including two species complexes: D. spathaceum (Rudolphi, 1819); D. pseudospathaceum Niewiadomska, 1984; D. parviventosum Dubois, 1932; three species-level lineages within the "D. baeri" species complex (Diplostomum sp. 'Lineages 3-5' sensu Blasco-Costa et al., 2014 [9]); three species-level lineages within the " $D$. mergi" species complex (Diplostomum sp. 'Lineages 2-4' sensu Georgieva et al., 2013 [7] and Selbach et al., 2015 [10]); Diplostomum sp. 'Clade Q' sensu Georgieva et al., 2013 [7]; and Diplostomum sp. 'Lineages 2 and 6' sensu Blasco-Costa et al., 2014 [9] (see [3, 7, 9, 10, 12, 16]).
However, although molecular data for metacercariae of Diplostomum spp. in fishes from European freshwater ecosystems have accumulated recently, most of the sequences originate from fish populations sampled in ponds and lakes in central and northern Europe (Germany, Iceland, Norway), and also predominantly from salmonid fishes. A single study provided molecular and morphological data for metacercariae of three species of Diplostomum spp. in endemic and invasive fish host species in Spain, at the southern distributional range of Diplostomum spp. in Europe [3]. However, no molecular data exist on species diversity and host ranges of these fish pathogens in large river systems in Europe.

Our study is the first to explore species diversity and host-parasite association patterns of Diplostomum spp. in a large riverine system in Europe. Here we extend the cox1 'barcode' reference library for Diplostomum spp. based on an extensive sampling of metacercariae from a broad range of fish hosts collected at two localities in the middle section of the River Danube. We provide molecular identification based on the cox 1 gene in association with a thorough morphological characterisation of the metacercariae. Further, we provide primers and the first assessment of the usefulness of the mitochondrial nicotinamide adenine dinucleotide dehydrogenase subunit 3 ( $n a d 3$ ) gene for species delineation within Diplostomum spp.

\section{Methods}

\section{Sample collection and processing}

A total of 174 fish belonging to 28 species of 9 families were sampled in the River Danube near Nyergesúffalu (47.7658N, 18.5417E) in Hungary in 2012 and at Štúrovo $(47.8197 \mathrm{~N}, 18.7286 \mathrm{E})$ in Slovakia in 2015. As a part of a complete helminthological examination, fish eyes and brains were isolated and examined for the presence of metacercariae of Diplostomum spp. The eyes were dissected and lens, vitreous humour and retina were placed in $0.9 \%$ saline solution and examined under a dissecting microscope. All metacercariae were collected and counted. Representative subsamples were selected for DNA isolation and sequencing. 


\section{Morphological examination}

The morphology of the metacercariae selected for sequencing was initially studied in live parasites; these were then transferred to molecular grade ethanol and re-examined. A series of photomicrographs was made for each isolate (live and fixed) using a digital camera of an Olympus BX51 microscope (Olympus Corporation, Tokyo, Japan). Measurements for each isolate were taken from the digital images with the aid of Quick Photo Camera 2.3 image analysis software. All measurements in the descriptions and tables are in micrometres and are presented as the range, followed by the mean in parentheses.

Fourteen morphometric variables were measured from the digital images of live and fixed metacercariae and the number of excretory concretions was recorded from live material. The following abbreviations for variables were used: BL, body length; BW, body width; HL, hindbody length; OSL, oral sucker length; OSW, oral sucker width; PSL, pseudosucker length; PSW, pseudosucker width; VSL, ventral sucker length; VSW, ventral sucker width; PHL, pharynx length; PHW, pharynx width; HOL, holdfast organ length; HOW, holdfast organ width; AVS, distance from anterior extremity of body to ventral sucker.

\section{Sequence generation}

Genomic DNA (gDNA) was isolated from single metacercariae using the E.Z.N.A. Tissue DNA Kit (Omega Bio-tek, Norcross, USA) following the manufacturer's instructions. Amplification of the mitochondrial (mt) $\operatorname{cox} 1$ gene was performed with the forward primer PlatdiploCOX1F (5'-CGT TTR AAT TAT ACG GAT CC$\left.3^{\prime}\right)$ and the reverse primer Plat-diploCOX1R (5'-AGC ATA GTA ATM GCA GCA GC-3') [4]. A pair of newly designed primers was used for amplification of the complete nad3 mt gene: forward Diplo-nad3F (5' -ATG TGA AAG TGG TGT TTG TT-3') and reverse Diplonad3R (5'-ATG CGC TTA TGA TCT AAC GT-3'). PCR amplifications for both genes were performed in a total volume of $20 \mu \mathrm{l}$ (8 pmol of each primer) with c. $50 \mathrm{ng}$ of gDNA and $10 \mu \mathrm{l}$ of $2 \times \mathrm{MyFi}^{\mathrm{Tm}}$ DNA Polymerase mix (Bioline Inc., Taunton, USA). Thermocycling started with an initial DNA denaturation for 2 min at $94{ }^{\circ} \mathrm{C}$ followed by 35 cycles with $30 \mathrm{~s}$ DNA denaturation at $94{ }^{\circ} \mathrm{C}$, $30 \mathrm{~s}$ primer annealing at $50{ }^{\circ} \mathrm{C}$ for $\operatorname{cox} 1\left(57^{\circ} \mathrm{C}\right.$ for $n a d 3$ ), and $60 \mathrm{~s}$ at $72{ }^{\circ} \mathrm{C}$ for primer extension, followed by a final extension step of $10 \mathrm{~min}$ at $72{ }^{\circ} \mathrm{C}$. PCR amplicons were purified using a QIAquick PCR purification kit (Qiagen Ltd., Hilden, Germany). Cycle sequencing of purified DNA was carried out using ABI Big Dye ${ }^{\text {Tu }}$ chemistry (ABI Perkin-Elmer, London, UK) on an Applied Biosystems 3730xl DNA Analyser following the manufacturer's recommendations, using the primers used for PCR amplification. Contiguous sequences were assembled with MEGA v6 [17] and submitted to GenBank under accession numbers KY653961-KY654066.

Unique $\operatorname{cox} 1$ haplotypes were identified with DnaSP [18] against all published sequences for a given species/ lineage. Unrooted statistical parsimony haplotype networks were constructed for D. spathaceum and D. pseudospathaceum using TCS 1.21 [19] with plausible branch connections between the haplotypes at a connection limit of $95 \%$ [20].

\section{Phylogenetic analyses}

Sequences were aligned using MUSCLE implemented in MEGA v6. Two alignments were analysed. The cox 1 alignment (410 nt) comprised 76 newly generated sequences and 31 sequences for Diplostomum spp. retrieved from GenBank; Tylodelphys clavata (von Nordmann, 1832) was used as the outgroup. The nad 3 alignment (357 nt) comprised 30 newly generated sequences and two published sequences, D. pseudospathaceum and D. spathaceum. Both alignments included no insertions or deletions and were aligned with reference to the amino acid translation, using the echinoderm and flatworm mitochondrial code [21]. Distance-based neighbour-joining (NJ) and modelbased Bayesian inference (BI) algorithms were conducted to identify and explore relationships among the species/ isolates. Neighbour-joining analyses of Kimura 2parameter distances were carried out using MEGA v6; nodal support was estimated using 1000 bootstrap resamplings. Bayesian inference analysis was performed for the cox1 dataset using MrBayes version 3.2.3 [22]. Prior to BI analysis, the best-fit nucleotide substitution model was selected in jModelTest 2.1.1 [23] using the Akaike Information Criterion (AIC). This was the general time reversible model, with estimates of invariant sites and gamma distributed among-site rate variation $(G T R+I+\Gamma)$. BI analysis was run with the following nucleotide substitution model settings: lset nst $=6$, rates $=$ invgamma, samplefreq $=100$, ncat $=4$, shape $=$ estimate, inferrates $=$ yes and basefreq $=$ empirical. Markov chain Monte Carlo (MCMC) chains were run for $10,000,000$ generations, log-likelihood scores were plotted and only the final $75 \%$ of trees were used to produce the consensus trees by setting the 'burn-in' parameter at 2500. Results were visualised in Tracer v.1.6 (http://tree.bio.ed.ac.uk/software/tracer/) to assess convergence and proper sampling and to identify the 'burn-in' period.

Distance matrices (uncorrected p-distance model) were calculated with MEGA v6. The nomenclature of Georgieva et al. [7] for the lineages of Diplostomum spp. was applied for consistency with previous records. 


\section{Results}

\section{General observations}

A total of 174 fish individuals belonging to 28 species and 9 families were examined for the presence of metacercariae of Diplostomum spp. in the eyes and brain. Only lensinfecting metacercariae were found in 16 fish species of 5 families: 12 cyprinids, one acipenserid, one lotid, one percid and one silurid (Table 1). The overall Diplostomum spp. intensity of infection was low (1-15 metacercariae per fish) with two exceptions: Abramis brama (25-43, four fishes) and Blicca bjoerkna (27, one fish). The overall Diplostomum spp. prevalence appeared rather high in five

Table 1 Summary data for the fish species examined/infected with Diplostomum spp.

\begin{tabular}{|c|c|c|c|}
\hline Host species & No. examined & No. infected & Diplostomum spp. \\
\hline \multicolumn{4}{|l|}{ Acipenseridae } \\
\hline Acipenser ruthenus L. & 1 & 1 & D. spathaceum \\
\hline \multicolumn{4}{|l|}{ Anguillidae } \\
\hline Anguilla anguilla (L.) & 1 & - & - \\
\hline \multicolumn{4}{|l|}{ Centrarchidae } \\
\hline Lepomis gibbosus (L.) & 11 & - & - \\
\hline \multicolumn{4}{|l|}{ Cyprinidae } \\
\hline Abramis brama (L.) & 41 & 34 & D. spathaceum, D. pseudospathaceum, 'D. mergi Lineage 2' \\
\hline Alburnus alburnus (L.) & 7 & 4 & 'D. mergi Lineage 2' \\
\hline Ballerus sapa (Pallas) & 9 & 2 & D. pseudospathaceum, 'D. mergi Lineage 2' \\
\hline Blicca bjoerkna (L.) & 13 & 10 & $\begin{array}{l}\text { D. spathaceum, D. pseudospathaceum, 'D. mergi Lineage 2', } \\
\text { Diplostomum sp. A }\end{array}$ \\
\hline Carassius gibelio (Bloch) & 6 & 1 & Diplostomum sp. B \\
\hline Chondrostoma nasus (L.) & 11 & 4 & D. spathaceum, 'D. mergi Lineage 2' \\
\hline Cyprinus carpio L. & 3 & 1 & D. pseudospathaceum \\
\hline Leuciscus aspius (L.) & 9 & 8 & D. spathaceum, D. pseudospathaceum \\
\hline Leuciscus idus (L.) & 4 & 1 & D. pseudospathaceum \\
\hline Rutilus pigus (Lacépède) & 3 & 2 & D. spathaceum \\
\hline Rutilus rutilus (L.) & 9 & 4 & D. spathaceum, Diplostomum sp. C \\
\hline Vimba vimba (L.) & 9 & 8 & D. spathaceum, D. pseudospathaceum, 'D. mergi Lineage 2' \\
\hline Barbus barbus (L.) & 2 & - & - \\
\hline Gobio gobio (L.) & 6 & - & - \\
\hline \multicolumn{4}{|l|}{ Esocidae } \\
\hline Esox lucius L. & 3 & - & - \\
\hline \multicolumn{4}{|l|}{ Gobiidae } \\
\hline Neogobius melanostomus (Pallas) & 8 & - & - \\
\hline Ponticola kessleri (Günther) & 2 & - & - \\
\hline \multicolumn{4}{|l|}{ Lotidae } \\
\hline Lota lota (L.) & 2 & 1 & D. pseudospathaceum \\
\hline \multicolumn{4}{|l|}{ Percidae } \\
\hline Gymnocephalus schraetser (L.) & 5 & 1 & D. pseudospathaceum \\
\hline Perca fluviatilis $\mathrm{L}$. & 3 & - & - \\
\hline Sander lucioperca (L.) & 1 & - & - \\
\hline Sander volgensis (Gmelin) & 2 & - & - \\
\hline Zingel zingel (L.) & 1 & - & - \\
\hline Zingel streber (Siebold) & 1 & - & - \\
\hline \multicolumn{4}{|l|}{ Siluridae } \\
\hline Silurus glanis L. & 1 & 1 & D. spathaceum \\
\hline
\end{tabular}


cyprinids (Leuciscus aspius: 89\%; Vimba vimba: 89\%; A. brama: 83\%; B. bjoerkna: 77\%; and Alburnus alburnus: $57 \%)$ but reliable estimates for prevalence could be obtained only for the sample of A. brama. In this sample, the prevalence of three species/lineages identified in our study (see below) was high: D. spathaceum: 75\%; 'D. mergi Lineage 2': 58\%; D. pseudospathaceum: 50\%. Twelve species of fish, for which fewer specimens were examined, were not infected.

Molecular identification, haplotype diversity and host-use We generated partial cox 1 sequences $(410 \mathrm{nt})$ for 76 isolates of Diplostomum spp. recovered from fishes of the River Danube (Table 2). These sequences were analysed together with 31 sequences for 10 Diplostomum species/ species-level genetic lineages retrieved from the GenBank database (see Additional file 1: Table S1 for details). All lens-infecting species/lineages of Diplostomum (7) reported in Europe were included in analyses: D. parviventosum, $D$. pseudospathaceum, D. spathaceum, 'D. mergi Lineage 2,' $D$. mergi Lineage 3', 'D. mergi Lineage 4, 'Diplostomum sp. Clade Q' sensu Georgieva et al., 2013 [7]. We also included sequences for D. huronense (a species believed to have a Holarctic distribution; see [24]) and two representatives of non-lens infecting species of the "D. baeri" complex. The branch topologies of the trees resulting from both, $\mathrm{NJ}$ and $\mathrm{BI}$ analyses, were in consensus in depicting species/species-level genetic lineages (Figs. 1, 2). The newly generated sequences clustered within three well-supported clades representing D. pseudospathaceum, D. spathaceum and ' $D$. mergi Lineage 2' except for three singletons which may potentially represent distinct species (labelled as Diplostomum sp. A, B and C in Fig. 2). Two of these (Diplostomum sp. $\mathrm{A}$ and $\mathrm{B}$ ) were resolved as basal to the clade representing the "D. mergi" species complex, whereas Diplostomum sp. C appeared associated with 'Clade Q'; however, these relationships were not supported.

The intraspecific divergence (uncorrected p-distance range), observed within the newly generated cox 1 sequences, ranged between 0 and $1.71 \%$ (mean $0.56 \%$ ) for D. pseudospathaceum, 0-1.95\% (mean 0.82\%) for $D$. spathaceum and $0-1.71 \%$ (mean $0.47 \%$ ) for ' $D$. mergi Lineage 2'. The three singletons exhibited high levels of divergence compared with the isolates of Diplostomum spp. included in the analyses: $7.1-15.6 \%$ for Diplostomum sp. A; $5.6-15.9 \%$ for Diplostomum sp. B; and 11.5$15.0 \%$ for Diplostomum sp. C.

The newly generated sequences for the three Diplostomum spp. were collapsed into 16 haplotypes for D. spathaceum, 15 haplotypes for D. pseudospathaceum and 7 haplotypes for ' $D$. mergi Lineage 2'. Of these, D. spathaceum and D. pseudospathaceum had 7 unique haplotypes each $(\mathrm{H} 1, \mathrm{H} 8, \mathrm{H} 9, \mathrm{H} 11$,
H14, H15, H16 and H3, H6, H8, H9, H11, H13, H14, respectively); and ' $D$. mergi Lineage 2' had 4 unique haplotypes ( $\mathrm{H} 3, \mathrm{H} 4, \mathrm{H} 5, \mathrm{H} 6)$.

Nine haplotypes of $D$. spathaceum were shared among isolates studied here and previously published sequences, predominantly generated in studies carried out in Europe (Germany, Iceland and Spain; see Georgieva et al. [7]; Pérez-del-Olmo et al. [3]; Selbach et al. [10]) (see Table 3 for details). Notably, four haplotypes ( $\mathrm{H} 2, \mathrm{H} 5$, $\mathrm{H} 6$ and H10) were shared between isolates from all three hosts in the species life-cycle (first intermediate hosts: Radix auricularia (L.) and Radix peregra (Müller); definitive hosts: Larus argentatus (s.l.) and L. ridibundus; second intermediate host: a number of fish species). Due to the geographical coverage of the previous studies, most of the shared haplotypes originate from Europe; however, sequence matches for isolates from Asia [6] indicate a wider distribution of six haplotypes (Iraq: H2, H5, H7 and H10; China: H2, H13) (Table 3). It is also worth noting that four of the haplotypes were shared with haplotypes implicated in a case of diplostomiasis in aquaculture of Pseudochondrostoma willkommii (Steindachner) [3].

Of the 15 haplotypes of $D$. pseudospathaceum, 8 were shared with previously reported isolates, predominantly from the first intermediate hosts, Lymnaea stagnalis (L.) and Stagnicola palustris (Müller), from the Czech Republic, Germany and Romania [6, 7, 10]; among these, a single haplotype (H2) was shared between isolates from all three hosts in the species life-cycle (Table 3 ). Finally, three haplotypes of ' $D$. mergi Lineage 2' were shared with isolates from the snail host $R$. auricularia in Germany (H1 and $\mathrm{H} 2$ ) and one with a metacercaria from A. brama in China (H7, see Table 3).

The cox 1 haplotype networks for $D$. spathaceum and D. pseudospathaceum, generated by statistical parsimony analysis, are presented in Figs. 3 and 4, respectively. For both species, haplotypes identified in the present material were sampled from 9 fish host species and there was no indication of genetic structuring associated with the host. The ancestral haplotype (H1) of D. spathaceum was recovered as unique and represented by isolates from 3 cyprinid hosts (A. brama, $R$. rutilus and $V$. vimba). Two other haplotypes ( $\mathrm{H} 2$ and $\mathrm{H} 3)$ were shared by isolates from 3 fish hosts each (A. brama, L. aspius and $R$. pigus and A. brama, R. pigus and S. glanis, respectively) (Fig. 3a). The cyprinid $A$. brama was the host with the largest haplotype diversity (8 haplotypes; 2 unique).

Figure 3b illustrates a haplotype network including all available sequence data for D. spathaceum from fish hosts in Europe and Asia. A total of 68 sequences was added for isolates from 12 fish species of five families: Cyprinidae (7 species; Locke et al. [6], Pérez-del-Olmo 
Table 2 Summary data for the isolates of Diplostomum spp. used for generation of the cox 1 and nad3 sequences

\begin{tabular}{|c|c|c|c|c|c|c|}
\hline \multirow[t]{2}{*}{ Species } & \multirow[t]{2}{*}{ Host } & \multirow[t]{2}{*}{ Country } & \multirow[t]{2}{*}{ Isolate } & \multirow[t]{2}{*}{ Haplotype (cox1) } & \multicolumn{2}{|c|}{ GenBank ID } \\
\hline & & & & & $\operatorname{cox} 1$ & nad3 \\
\hline D. spathaceum & Abramis brama & $S$ & ABD1 & $\mathrm{H} 11$ & KY653961 & KY654037 \\
\hline D. spathaceum & Abramis brama & S & ABD2 & $\mathrm{H} 1$ & KY653962 & \\
\hline D. spathaceum & Abramis brama & S & ABD3 & $\mathrm{H} 1$ & KY653963 & \\
\hline D. spathaceum & Abramis brama & S & ABD4 & $\mathrm{H} 5$ & KY653964 & \\
\hline D. spathaceum & Abramis brama & S & ABD5 & $\mathrm{H} 9$ & KY653965 & \\
\hline D. spathaceum & Abramis brama & S & ABD6 & $\mathrm{H} 12$ & KY653966 & KY654038 \\
\hline D. spathaceum & Abramis brama & S & ABD7 & $\mathrm{H} 10$ & KY653967 & \\
\hline D. spathaceum & Abramis brama & S & ABD8 & $\mathrm{H} 2$ & KY653968 & \\
\hline D. spathaceum & Abramis brama & S & ABD9 & $\mathrm{H} 3$ & KY653969 & KY654039 \\
\hline D. spathaceum & Acipenser ruthenus & S & ARD & $\mathrm{H} 4$ & KY653970 & \\
\hline D. spathaceum & Blicca bjoerkna & S & BBD1 & $\mathrm{H} 6$ & KY653971 & \\
\hline D. spathaceum & Blicca bjoerkna & S & BBD2 & $\mathrm{H} 4$ & KY653972 & KY654040 \\
\hline D. spathaceum & Blicca bjoerkna & $\mathrm{H}$ & BBD3 & $\mathrm{H} 14$ & KY653973 & \\
\hline D. spathaceum & Chondrostoma nasus & $S$ & CND1 & $\mathrm{H} 7$ & KY653974 & KY654041 \\
\hline D. spathaceum & Chondrostoma nasus & $\mathrm{H}$ & CND2 & $\mathrm{H} 15$ & KY653975 & \\
\hline D. spathaceum & Leuciscus aspius & $\mathrm{H}$ & LAD1 & $\mathrm{H} 13$ & KY653976 & KY654042 \\
\hline D. spathaceum & Leuciscus aspius & S & LAD2 & $\mathrm{H} 2$ & KY653977 & \\
\hline D. spathaceum & Rutilus pigus & S & RPD1 & $\mathrm{H} 5$ & KY653978 & \\
\hline D. spathaceum & Rutilus pigus & S & RPD2 & $\mathrm{H} 2$ & KY653979 & KY654043 \\
\hline D. spathaceum & Rutilus pigus & S & RPD3 & $\mathrm{H} 8$ & KY653980 & \\
\hline D. spathaceum & Rutilus pigus & S & RPD4 & $\mathrm{H} 3$ & KY653981 & KY654044 \\
\hline D. spathaceum & Rutilus rutilus & S & RRD1 & $\mathrm{H} 1$ & KY653982 & KY654045 \\
\hline D. spathaceum & Rutilus rutilus & $\mathrm{H}$ & RRD2 & $\mathrm{H} 16$ & KY653983 & \\
\hline D. spathaceum & Silurus glanis & S & $S G D$ & $\mathrm{H} 3$ & KY653984 & KY654046 \\
\hline D. spathaceum & Vimba vimba & S & WD1 & $\mathrm{H} 1$ & KY653985 & \\
\hline D. spathaceum & Vimba vimba & S & WD2 & $\mathrm{H} 1$ & KY653986 & \\
\hline D. pseudospathaceum & Abramis brama & S & ABD10 & $\mathrm{H} 1$ & KY653987 & KY654047 \\
\hline D. pseudospathaceum & Abramis brama & S & ABD11 & $\mathrm{H} 1$ & KY653988 & \\
\hline D. pseudospathaceum & Abramis brama & S & $\mathrm{ABD} 12$ & $\mathrm{H} 2$ & KY653989 & KY654048 \\
\hline D. pseudospathaceum & Abramis brama & S & ABD13 & $\mathrm{H} 14$ & KY653990 & \\
\hline D. pseudospathaceum & Abramis brama & S & ABD14 & $\mathrm{H} 15$ & KY653991 & \\
\hline D. pseudospathaceum & Ballerus sapa & S & BSD1 & $\mathrm{H} 1$ & KY653992 & KY654049 \\
\hline D. pseudospathaceum & Ballerus sapa & $S$ & BSD2 & $\mathrm{H} 3$ & KY653993 & KY654050 \\
\hline D. pseudospathaceum & Ballerus sapa & S & BSD3 & $\mathrm{H} 3$ & KY653994 & \\
\hline D. pseudospathaceum & Ballerus sapa & S & BSD4 & $\mathrm{H} 2$ & KY653995 & \\
\hline D. pseudospathaceum & Blicca bjoerkna & $\mathrm{H}$ & BBD4 & $\mathrm{H} 1$ & KY653996 & \\
\hline D. pseudospathaceum & Blicca bjoerkna & S & BBD5 & $\mathrm{H} 7$ & KY653997 & KY654051 \\
\hline D. pseudospathaceum & Blicca bjoerkna & S & BBD6 & $\mathrm{H} 8$ & KY653998 & KY654052 \\
\hline D. pseudospathaceum & Blicca bjoerkna & S & BBD7 & $\mathrm{H} 10$ & KY653999 & \\
\hline D. pseudospathaceum & Blicca bjoerkna & S & BBD8 & $\mathrm{H} 11$ & KY654000 & \\
\hline D. pseudospathaceum & Blicca bjoerkna & $\mathrm{H}$ & BBD9 & $\mathrm{H} 4$ & KY654001 & \\
\hline D. pseudospathaceum & Blicca bjoerkna & $\mathrm{s}$ & BBD10 & $\mathrm{H} 9$ & KY654002 & \\
\hline
\end{tabular}


Table 2 Summary data for the isolates of Diplostomum spp. used for generation of the cox 1 and nad3 sequences (Continued)

\begin{tabular}{|c|c|c|c|c|c|c|}
\hline \multirow[t]{2}{*}{ Species } & \multirow[t]{2}{*}{ Host } & \multirow[t]{2}{*}{ Country } & \multirow[t]{2}{*}{ Isolate } & \multirow[t]{2}{*}{ Haplotype (cox1) } & \multicolumn{2}{|c|}{ GenBank ID } \\
\hline & & & & & $\cos 1$ & nad3 \\
\hline D. pseudospathaceum & Cyprinus carpio & S & $C C D$ & $\mathrm{H} 1$ & KY654003 & KY654053 \\
\hline D. pseudospathaceum & Gymnocephalus schraetser & $\mathrm{H}$ & GSD & $\mathrm{H} 4$ & KY654004 & \\
\hline D. pseudospathaceum & Leuciscus aspius & S & LAD3 & $\mathrm{H} 13$ & KY654005 & \\
\hline D. pseudospathaceum & Leuciscus aspius & $S$ & LAD4 & $\mathrm{H} 1$ & KY654006 & \\
\hline D. pseudospathaceum & Leuciscus aspius & S & LAD5 & $\mathrm{H} 2$ & KY654007 & \\
\hline D. pseudospathaceum & Leuciscus aspius & $S$ & LAD6 & $\mathrm{H} 6$ & KY654008 & \\
\hline D. pseudospathaceum & Leuciscus aspius & S & LAD7 & $\mathrm{H} 5$ & KY654009 & KY654054 \\
\hline D. pseudospathaceum & Leuciscus aspius & S & LAD8 & $\mathrm{H} 5$ & KY654010 & \\
\hline D. pseudospathaceum & Leuciscus aspius & $\mathrm{H}$ & LAD9 & $\mathrm{H} 4$ & KY654011 & \\
\hline D. pseudospathaceum & Leuciscus idus & S & LID1 & $\mathrm{H} 1$ & KY654012 & KY654055 \\
\hline D. pseudospathaceum & Leuciscus idus & $S$ & LID2 & $\mathrm{H} 12$ & KY654013 & \\
\hline D. pseudospathaceum & Lota lota & $\mathrm{H}$ & LLD & $\mathrm{H} 3$ & KY654014 & \\
\hline D. pseudospathaceum & Vimba vimba & $S$ & WD3 & $\mathrm{H} 1$ & KY654015 & KY654056 \\
\hline D. pseudospathaceum & Vimba vimba & $\mathrm{H}$ & WD4 & $\mathrm{H} 1$ & KY654016 & \\
\hline 'D. mergi Lineage 2' & Abramis brama & $S$ & ABD15 & $\mathrm{H} 2$ & KY654017 & \\
\hline 'D. mergi Lineage 2' & Abramis brama & S & ABD16 & $\mathrm{H} 4$ & KY654018 & KY654057 \\
\hline 'D. mergi Lineage 2' & Abramis brama & S & ABD17 & $\mathrm{H} 1$ & KY654019 & KY654058 \\
\hline 'D. mergi Lineage 2' & Abramis brama & S & ABD18 & $\mathrm{H} 2$ & KY654020 & KY654059 \\
\hline 'D. mergi Lineage 2' & Alburnus alburnus & $\mathrm{H}$ & AAD1 & $\mathrm{H} 2$ & KY654021 & \\
\hline 'D. mergi Lineage 2' & Alburnus alburnus & $S$ & AAD2 & H5 & KY654022 & KY654060 \\
\hline 'D. mergi Lineage 2' & Alburnus alburnus & $\mathrm{H}$ & AAD3 & $\mathrm{H} 1$ & KY654023 & KY654061 \\
\hline 'D. mergi Lineage 2' & Alburnus alburnus & $\mathrm{H}$ & AAD4 & $\mathrm{H} 1$ & KY654024 & \\
\hline 'D. mergi Lineage 2' & Alburnus alburnus & $\mathrm{H}$ & AAD5 & $\mathrm{H} 1$ & KY654025 & \\
\hline 'D. mergi Lineage 2' & Alburnus alburnus & $\mathrm{H}$ & AAD6 & $\mathrm{H} 1$ & KY654026 & \\
\hline 'D. mergi Lineage 2' & Ballerus sapa & $\mathrm{H}$ & BSD5 & $\mathrm{H} 7$ & KY654027 & KY654062 \\
\hline 'D. mergi Lineage 2' & Blicca bjoerkna & $S$ & BBD11 & $\mathrm{H} 3$ & KY654028 & KY654063 \\
\hline 'D. mergi Lineage 2' & Blicca bjoerkna & $S$ & $\mathrm{BBD} 12$ & $\mathrm{H} 1$ & KY654029 & KY654064 \\
\hline 'D. mergi Lineage 2' & Blicca bjoerkna & $\mathrm{H}$ & BBD13 & $\mathrm{H} 1$ & KY654030 & \\
\hline 'D. mergi Lineage 2' & Chondrostoma nasus & $S$ & CND3 & $\mathrm{H} 1$ & KY654031 & KY654065 \\
\hline 'D. mergi Lineage 2' & Vimba vimba & $\mathrm{H}$ & WD5 & $\mathrm{H} 6$ & KY654032 & \\
\hline 'D. mergi Lineage 2' & Vimba vimba & $\mathrm{H}$ & WD6 & $\mathrm{H} 1$ & KY654033 & KY654066 \\
\hline Diplostomum sp. A & Blicca bjoerkna & S & BBD14 & - & KY654034 & \\
\hline Diplostomum sp. B & Carassius gibelio & S & CGD & - & KY654035 & \\
\hline Diplostomum sp. C & Rutilus rutilus & S & RRD3 & - & KY654036 & \\
\hline
\end{tabular}

Abbreviations: $H$ Hungary, S Slovakia

et al. [3]); Gasterosteidae (1 species; Georgieva et al. [7], Blasco-Costa et al. [9]); Cobitidae (1 species; Pérez-delOlmo et al. [3]); Percidae (1 species; Locke et al. [6]); Salmonidae (1 species; Blasco-Costa et al. [9]) and Siluridae (1 species; Locke et al. [6]) (see Additional file 2: Table S2 for details). This expanded dataset comprising 94 sequences (trimmed to $402 \mathrm{nt}$ ) for isolates from 17 fish host species of 7 families revealed a much higher haplotype diversity (55 haplotypes) and a generally similar pattern for the most common haplotypes. However, a large number of haplotypes were represented by singletons (45 haplotypes: H8, H9, H11, H14-H55, see Additional file 2: Table S2) and H2 was the most common haplotype in the expanded network. A total of 30 haplotypes was identified in isolates sampled recently in China $(n=4)$ and Iraq $(n=26)$ by Locke et al. [6], and 


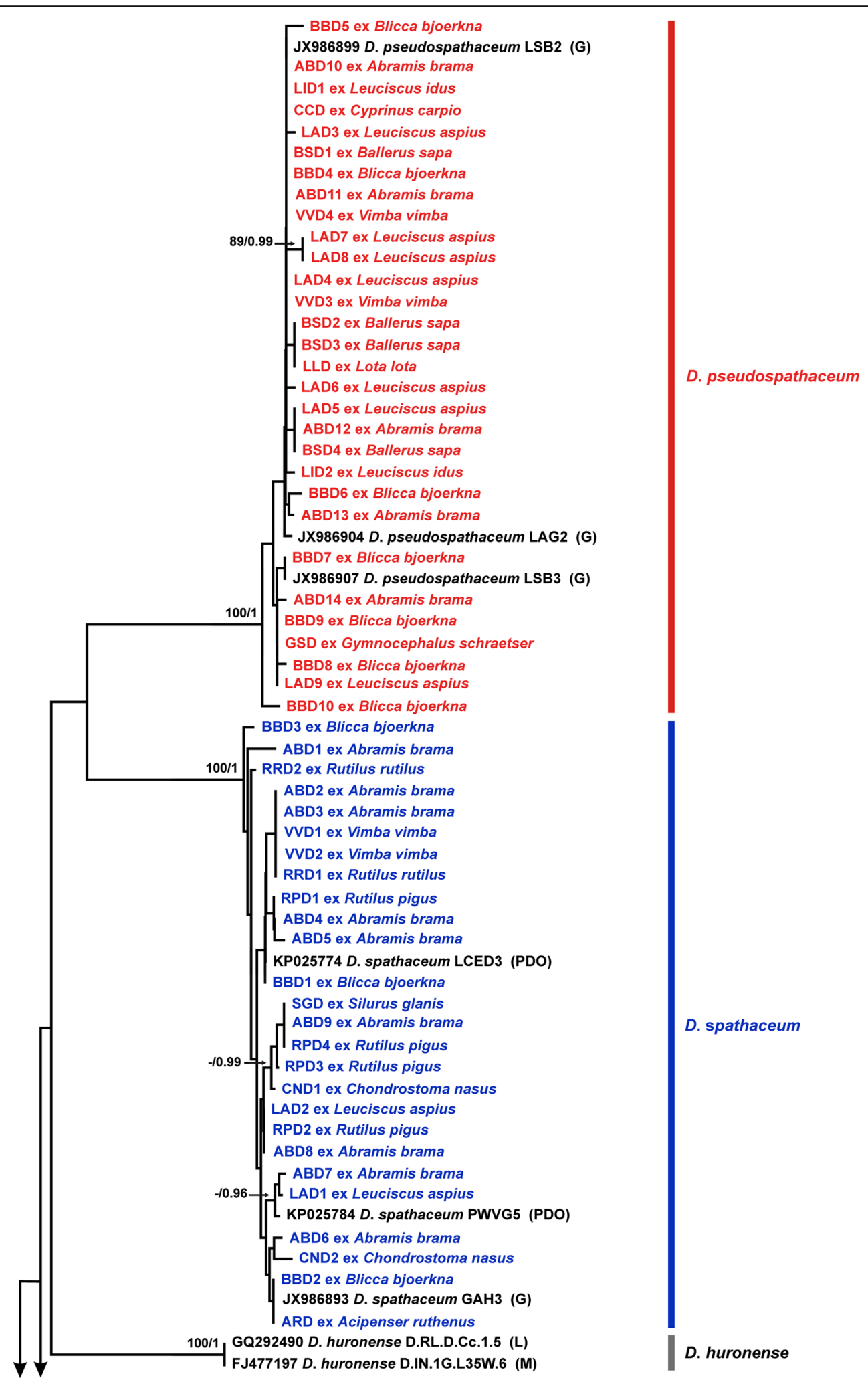

Fig. 1 Neighbour-joining (NJ) phylogram for Diplostomum spp. reconstructed using 76 newly generated and 31 cox 1 sequences retrieved from GenBank. Outgroup: Tylodelphys clavata. Nodal support from NJ and Bayesian inference (BI) analyses are indicated as NJ/Bl; only values $>70 \%(\mathrm{NJ})$ and $>0.95$ (BI) are shown. The scale-bar indicates the expected number of substitutions per site. Codes for the newly sequenced isolates are provided in Table 2 . Sequence identification is as in GenBank, followed by a letter: G, Georgieva et al. [7]; L, Locke et al. [5]; M, Moszczynska et al. [4]; PDO, Pérez-del-Olmo et al. [3] 


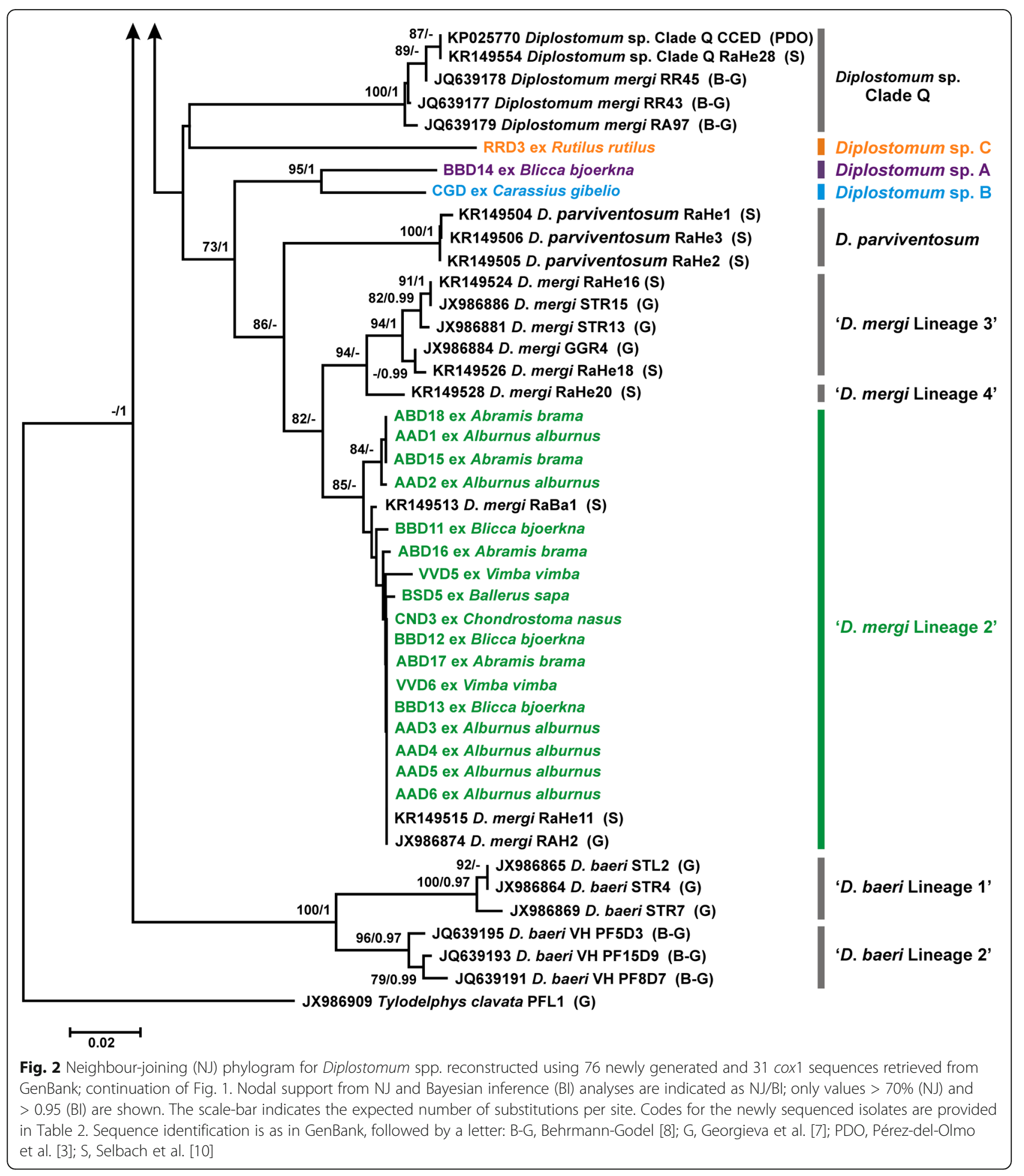

five haplotypes $(\mathrm{H} 2, \mathrm{H} 5, \mathrm{H} 7, \mathrm{H} 10$ and $\mathrm{H} 13)$ were shared by isolates from Europe and Asia (Fig. 3b; Table 3). Notably, three of the five major haplotypes (H2-H4) recovered from different host species in the River Danube (Fig. 3a) also exhibited low host-specificity at the level of host family (associated with fish hosts of 2-5 families, see Fig. 3b) whereas haplotypes $\mathrm{H} 1$ and $\mathrm{H} 5$ appear to be restricted to the Cyprinidae based on the currently available data.

Diplostomum pseudospathaceum exhibited a marked contrast in haplotype network structure (star-shaped network, indicative of range expansion, see Fig. 4a) 
Table 3 Details for the hosts, localities and GenBank accession numbers for the shared haplotypes of Diplostomum spp. identified in fishes from the River Danube

\begin{tabular}{|c|c|c|c|c|c|c|}
\hline \multirow[t]{2}{*}{ Species/Haplotype } & \multicolumn{2}{|l|}{ Present study } & \multicolumn{4}{|c|}{ Published isolates with matching sequences } \\
\hline & Isolate code ${ }^{a}$ & Host & GenBank ID & Host & Origin & Reference \\
\hline \multicolumn{7}{|c|}{ Diplostomum spathaceum } \\
\hline $\mathrm{H} 2$ & ABD8; LAD2; RPD2 & $\begin{array}{l}\text { A. brama; L. aspius; } \\
\text { R. pigus }\end{array}$ & $\begin{array}{l}\text { JX986889; KR149550; } \\
\text { KR149553; JX986888; } \\
\text { KJ726433, KJ726434; } \\
\text { KR271463; KR271451; } \\
\text { KR271426; KR271430; } \\
\text { JX986887 }\end{array}$ & $\begin{array}{l}\text { Snails: Radix auricularia } \\
\text { Fishes: Abramis brama; } \\
\text { Acanthobrama marmid; } \\
\text { Barbus luteus; Cyprinion } \\
\text { macrostomum; Gasterosteus } \\
\text { aculeatus } \\
\text { Birds: Larus cachinnans }\end{array}$ & $\begin{array}{l}\text { China; Czech } \\
\text { Republic; Germany; } \\
\text { Iceland; Iraq }\end{array}$ & {$[6,7,9,10]$} \\
\hline $\mathrm{H} 3$ & ABD9; RPD4; SGD & $\begin{array}{l}\text { A. brama; R. pigus; } \\
\text { S. glanis }\end{array}$ & JX986894; KR271417 & $\begin{array}{l}\text { Fishes: Gasterosteus } \\
\text { aculeatus; Perca fluviatilis }\end{array}$ & Germany; Italy & {$[6,7]$} \\
\hline $\mathrm{H} 4$ & ARD; BBD2 & $\begin{array}{l}\text { A. ruthenus; } \\
\text { B. bjoerkna }\end{array}$ & $\begin{array}{l}\text { JX986893; KP025775; } \\
\text { KP025785; KJ726438; } \\
\text { KR271462 }\end{array}$ & $\begin{array}{l}\text { Fishes: Gasterosteus } \\
\text { aculeatus; } \\
\text { Pseudochondrostoma } \\
\text { willkommii; Salvelinus } \\
\text { alpinus; Silurus glanis } \\
\text { Birds: Larus ridibundus }\end{array}$ & $\begin{array}{l}\text { Germany; Iceland; } \\
\text { Romania; Spain }\end{array}$ & {$[3,6,7,9]$} \\
\hline $\mathrm{H} 5$ & ABD4; RPD1 & A. brama; R. pigus & $\begin{array}{l}\text { JX986892; KR149551; } \\
\text { KR271422, KR271429; } \\
\text { KP025783; KP025772 }\end{array}$ & $\begin{array}{l}\text { Snails: Radix auricularia. } \\
\text { Fishes: } \\
\text { Cyprinion macrostomum; } \\
\text { Pseudochondrostoma } \\
\text { willkommii } \\
\text { Birds: Larus argentatus; L. } \\
\text { argentatus michahellis }\end{array}$ & $\begin{array}{l}\text { Germany; Iraq; } \\
\text { Poland; Spain }\end{array}$ & {$[3,6,7,10]$} \\
\hline $\mathrm{H} 6$ & BBD1 & B. bjoerkna & $\begin{array}{l}\text { KR149547, KR149548; } \\
\text { KP025781; KP025778; } \\
\text { KP025774; KJ726435; } \\
\text { KJ726436; KR271431 }\end{array}$ & $\begin{array}{l}\text { Snails: Radix auricularia; } \\
\text { Radix peregra } \\
\text { Fishes: Gasterosteus aculeatus; } \\
\text { Misgurnus anguillicaudatus; } \\
\text { Pseudochondrostoma } \\
\text { willkommii } \\
\text { Birds: Larus argentatus } \\
\text { michahellis }\end{array}$ & $\begin{array}{l}\text { Germany; Iceland; } \\
\text { Spain }\end{array}$ & {$[3,6,9,10]$} \\
\hline $\mathrm{H} 7$ & CND1 & C. nasus & $\begin{array}{l}\text { JX986891; KR149552; } \\
\text { JX986890; KP025786; } \\
\text { KP025782; KR271452; } \\
\text { KR271423 }\end{array}$ & $\begin{array}{l}\text { Snails: Radix auricularia } \\
\text { Fishes: Acanthobrama } \\
\text { marmid; Cyprinion } \\
\text { macrostomum; Gasterosteus } \\
\text { aculeatus; } \\
\text { Pseudochondrostoma } \\
\text { willkommii }\end{array}$ & $\begin{array}{l}\text { Germany; Iraq; } \\
\text { Spain }\end{array}$ & {$[3,6,7,10]$} \\
\hline $\mathrm{H} 10$ & ABD7 & A. brama & $\begin{array}{l}\text { KR149549; KP025779; } \\
\text { KR271428; JX986895 }\end{array}$ & $\begin{array}{l}\text { Snails: Radix auricularia } \\
\text { Fishes: Barbus luteus; } \\
\text { Misgurnus anguillicaudatus } \\
\text { Birds: Larus cachinnans }\end{array}$ & $\begin{array}{l}\text { Germany; Iraq; } \\
\text { Poland; Spain }\end{array}$ & {$[3,6,7,10]$} \\
\hline $\mathrm{H} 12$ & ABD6 & A. brama & KR271420 & Fishes: Perca fluviatilis & Italy & {$[6]$} \\
\hline $\mathrm{H} 13$ & LAD1 & L. aspius & KR271459 & Fishes: Abramis brama & China & [6] \\
\hline \multicolumn{7}{|c|}{ Diplostomum pseudospathaceum } \\
\hline $\mathrm{H} 1$ & $\begin{array}{l}\text { ABD10; ABD11; BBD4; } \\
\text { BSD1; CCD; LAD4; } \\
\text { LID1; WD3; WD4 }\end{array}$ & $\begin{array}{l}\text { A. brama; } B . \\
\text { bjoerkna; B. sapa; C. } \\
\text { carpio; L. aspius; L. } \\
\text { idus; V. vimba }\end{array}$ & $\begin{array}{l}\text { JX986899; JX986900; } \\
\text { KR149529; KR149535; } \\
\text { KR149536; KR271088; } \\
\text { JX986901; KR271090; } \\
\text { KR271091 }\end{array}$ & $\begin{array}{l}\text { Snails: Lymnaea stagnalis; } \\
\text { Stagnicola palustris } \\
\text { Fishes: Silurus glanis }\end{array}$ & Germany; Romania & {$[6,7,10]$} \\
\hline $\mathrm{H} 2$ & ABD12; BSD4; LAD5 & $\begin{array}{l}\text { A. brama; B. sapa; } \\
\text { L. aspius }\end{array}$ & $\begin{array}{l}\text { JX986897; KR149534; } \\
\text { KR149533; KR149532; } \\
\text { KR149530; JX986898; } \\
\text { KR149541; KR271093; } \\
\text { JX986896 }\end{array}$ & $\begin{array}{l}\text { Snails: Lymnaea stagnalis; } \\
\text { Stagnicola palustris } \\
\text { Fishes: Cyprinus carpio } \\
\text { Birds: Larus cachinnans }\end{array}$ & $\begin{array}{l}\text { Czech Republic; } \\
\text { Germany; Romania }\end{array}$ & {$[6,7,10]$} \\
\hline $\mathrm{H} 4$ & BBD9; GSD; LAD9 & $\begin{array}{l}\text { B. bjoerkna; G. } \\
\text { schraetsor; } L \text {. } \\
\text { aspius }\end{array}$ & KR149546 & Snails: Stagnicola palustris & Germany & {$[10]$} \\
\hline H5 & LAD7; LAD8 & L. aspius & JX986902; JX986903 & Fishes: Gasterosteus aculeatus & Germany & {$[7]$} \\
\hline $\mathrm{H} 7$ & BBD5 & B. bjoerkna & KR149542 & Snails: Stagnicola palustris & Germany & {$[10]$} \\
\hline $\mathrm{H} 10$ & BBD7 & B. bjoerkna & JX986907 & Snails: Lymnaea stagnalis & Germany & {$[7]$} \\
\hline $\mathrm{H} 12$ & LID2 & L. idus & KR149531 & Snails: Lymnaea stagnalis & Germany & {$[10]$} \\
\hline $\mathrm{H} 15$ & $\mathrm{ABD} 14$ & A. brama & KR149537 & Snails: Stagnicola palustris & Germany & {$[10]$} \\
\hline
\end{tabular}


Table 3 Details for the hosts, localities and GenBank accession numbers for the shared haplotypes of Diplostomum spp. identified in fishes from the River Danube (Continued)

\begin{tabular}{|c|c|c|c|c|c|c|}
\hline \multirow[t]{2}{*}{ Species/Haplotype } & \multicolumn{2}{|l|}{ Present study } & \multicolumn{4}{|c|}{ Published isolates with matching sequences } \\
\hline & Isolate code ${ }^{a}$ & Host & GenBank ID & Host & Origin & Reference \\
\hline \multicolumn{7}{|c|}{ 'Diplostomum mergi Lineage 2' } \\
\hline $\mathrm{H} 1$ & $\begin{array}{l}\text { AAD3; AAD4; AAD5; } \\
\text { AAD6; ABD17; BBD12; } \\
\text { BBD13; CND3; WD6 }\end{array}$ & $\begin{array}{l}\text { A. alburnus; A. brama; } \\
\text { B. bjoerkna; C. nasus; } \\
\text { V. vimba }\end{array}$ & $\begin{array}{l}\text { JX986874; JX986875; } \\
\text { JX986876; KR149522; } \\
\text { KR149521; KR149520; } \\
\text { KR149518; KR149517; } \\
\text { KR149515; KR149514 }\end{array}$ & Snails: Radix auricularia & Germany & {$[7,10]$} \\
\hline $\mathrm{H} 2$ & AAD1; ABD15; ABD18 & A. alburnus; A. brama & $\begin{array}{l}\text { KR149523; KR149519; } \\
\text { KR149516 }\end{array}$ & Snails: Radix auricularia & Germany & {$[10]$} \\
\hline $\mathrm{H} 7$ & BSD5 & B. sapa & KR271082 & Fishes: Abramis brama & China & [6] \\
\hline
\end{tabular}

${ }^{\mathrm{a} S e e}$ Table 2 for details

compared to the more complex network for D. spathaceum. The ancestral haplotype (H1) was shared among isolates from 7 of the 9 fish hosts (all cyprinids). The largest haplotype diversity was also found in cyprinid fishes: B. bjoerkna (7 haplotypes; 3 unique) followed by $L$. aspius (6 haplotypes, 2 unique). The haplotype network, including all available sequence data for D. pseudospathaceum from fish hosts in Europe (Fig. 4b) (12 host species of 5 families), includes 11 additional sequences for isolates from 3 fish species of 3 families: Cyprinidae (2 species; Locke et al. [6]); Gasterosteidae (1 species; Georgieva et al. [7]); and Siluridae (1 species; Locke et al. [6]) (see Additional file 2: Table S2 for details). This resulted in adding 6 new haplotypes (all singletons) to the dataset (41 sequences, trimmed to 402 nt; 21 haplotypes, see Additional file 2: Table S2). The haplotype network (Fig. 4b) closely resembled that for fishes sampled in the River Danube (Fig. 4a). Three of the four haplotypes identified in isolates from different fish species in the River Danube were also recovered in non-cyprinid fishes (Fig. 4b) (H1: Siluridae; H3: Lotidae; and H4: Percidae) and one haplotype (H5) was also identified in isolates from G. aculeatus (Gasterosteidae) (Georgieva et al. [7]).

To aid further exploration of species boundaries among the most widespread lens-infecting Diplostomum spp., the nad3 gene was selected based on its lower level of sequence conservation (83.3\%) compared with the 'barcode' region of the cox 1 gene (90.6\%) (see Brabec et al. [25]). A total of 30 complete nad 3 sequences (357 nt) were generated for the three species identified based on the cox 1 gene subsampling (10 isolates per species; see Table 2 for details). NJ analysis of the nad3 dataset depicted three distinct well-supported monophyletic clades corresponding to the cox1 lineages (Fig. 5). The levels of the interspecific divergence for the nad3 gene was distinctly higher with minimum $\mathrm{p}$-distance values well above the maximum values for cox 1 (14.6-15.7 vs 9-11.2\%) (Table 4). It is worth noting that the use of the newly designed primers resulted in successful amplification of nad3 in the distantly related lineage of the "D. mergi" complex of cryptic species.

\section{Descriptions of the molecular voucher material}

Comparisons based on live metacercariae of the most frequent species in this study, D. spathaceum, D. pseudospathaceum and ' $D$. mergi Lineage 2' revealed that metacercariae of $D$. spathaceum exhibit the highest mean values for the width of the body, the length of the hindbody, and the size of the oral sucker, pseudosuckers and pharynx. Live metacercariae of $D$. pseudospathaceum were characterised by the lowest mean values for the size of the body, pseudosuckers and holdfast organ whereas those of ' $D$. mergi Lineage 2' exhibited the highest mean values for the length of the body and the size of the ventral sucker and holdfast organ. Surprisingly, fixed metacercariae of ' $D$. mergi Lineage 2' demonstrated the highest mean values for the size of the body, pseudosuckers, ventral sucker, holdfast organ and hindbody whereas the dimensions of specimens of $D$. spathaceum and D. pseudospathaceum were rather similar (see Tables 5, 6). We have therefore provided morphological and morphometric characterisation based on both live and fixed material.

Unfortunately, the single metacercariae of Diplostomum sp. A, Diplostomum sp. B and Diplostomum sp. C were fixed in the field and their descriptions are based on fixed material. Nevertheless, comparisons based on fixed metacercariae of the six forms recovered in the present study indicate that the sucker ratios and the number and relative size of the excretory concretions are the most prominent characters that can be used for their discrimination. Diplostomum sp. A and B exhibited the largest values for the sucker width ratio and were characterised by having large excretory concretions, similar to those observed in D. spathaceum. However, the metacercaria of Diplostomum sp. B is much larger $(426 \times 304 v s$ a mean of $346 \times 288 \mu \mathrm{m}$ for $D$. spathaceum) and the excretory concretions in the metacercaria of Diplostomum sp. A also appear larger than in the 


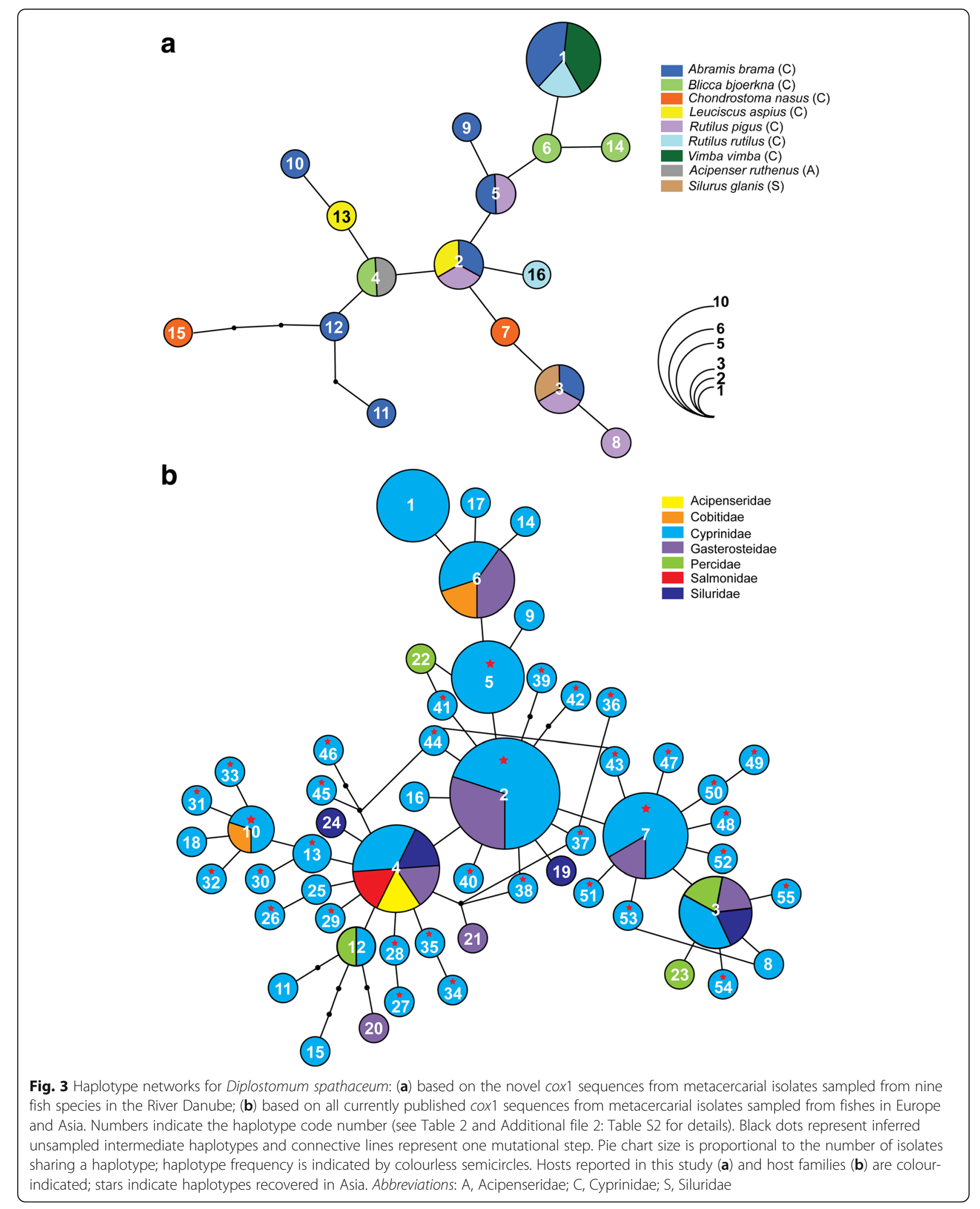




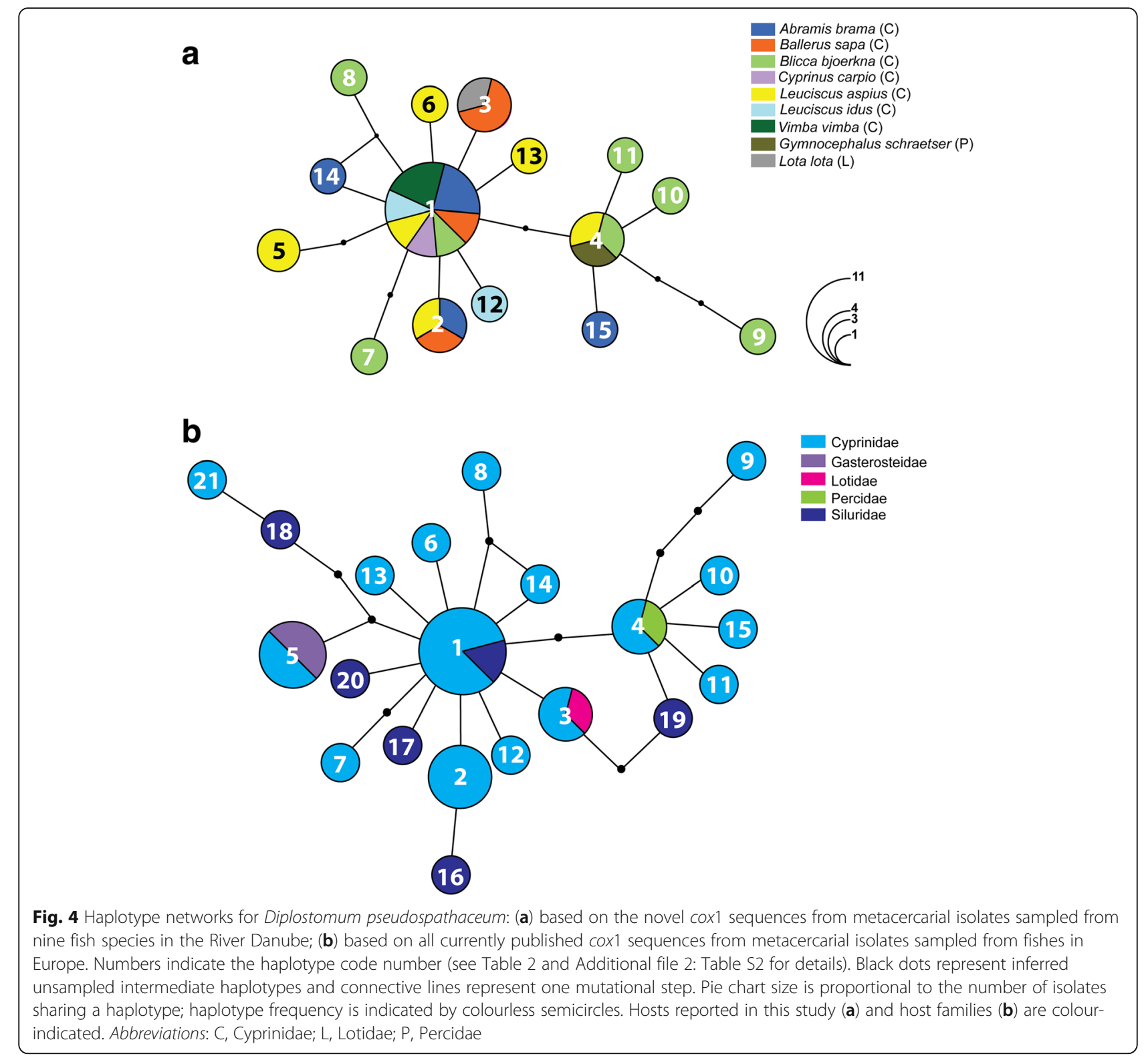

metacercaria of D. spathaceum (Fig. 6). The metacercaria of Diplostomum sp. C can be distinguished from the other five forms in having the largest number of excretory concretions (482 vs a maximum of 254, 360, 440 in D. spathaceum, D. pseudospathaceum and 'Diplostomum mergi Lineage 2', respectively, and 154 and 261 in Diplostomum sp. A and Diplostomum sp. B, respectively) (see also Fig. 6).

\section{Diplostomum spathaceum (Rudolphi, 1819)}

Hosts: Acipenser ruthenus L. (Chondrostei: Acipenseridae), Abramis brama (L.), Blicca bjoerkna (L.), Chondrostoma nasus (L.), Leuciscus aspius (L.), Rutilus pigus
(Lacépède), Rutilus rutilus (L.), Vimba vimba (L.) (Teleostei: Cyprinidae); Silurus glanis L. (Teleostei: Siluridae). Prevalence: $A$. ruthenus: 1/1 (Slovakia, S); A. brama: 75\% (29/40, S); B. bjoerkna: 1/5 (Hungary, H), 1/8 (S); C. nasus: $2 / 7(\mathrm{H}), 1 / 5(\mathrm{~S}) ;$ L. aspius: $3 / 6(\mathrm{H}), 1 / 4(\mathrm{~S}) ; R$. pigus: $2 / 3(\mathrm{~S}) ;$ R. rutilus: $1 / 1(\mathrm{H}), 2 / 8(\mathrm{~S}) ;$ V. vimba: $2 / 4$ (S); S. glanis: 1/1 (S).

Representative DNA sequences: KY653961-KY653986 (cox1); KY654037-KY654046 (nad3).

\section{Description}

[Based on 20 live metacercariae. Metrical data for fixed material are provided in Table 5; Fig. 6a.] Body oval, $349-601 \times 265-442(474 \times 341)$, with maximum width just anterior to ventral sucker. Oral sucker elongate- 


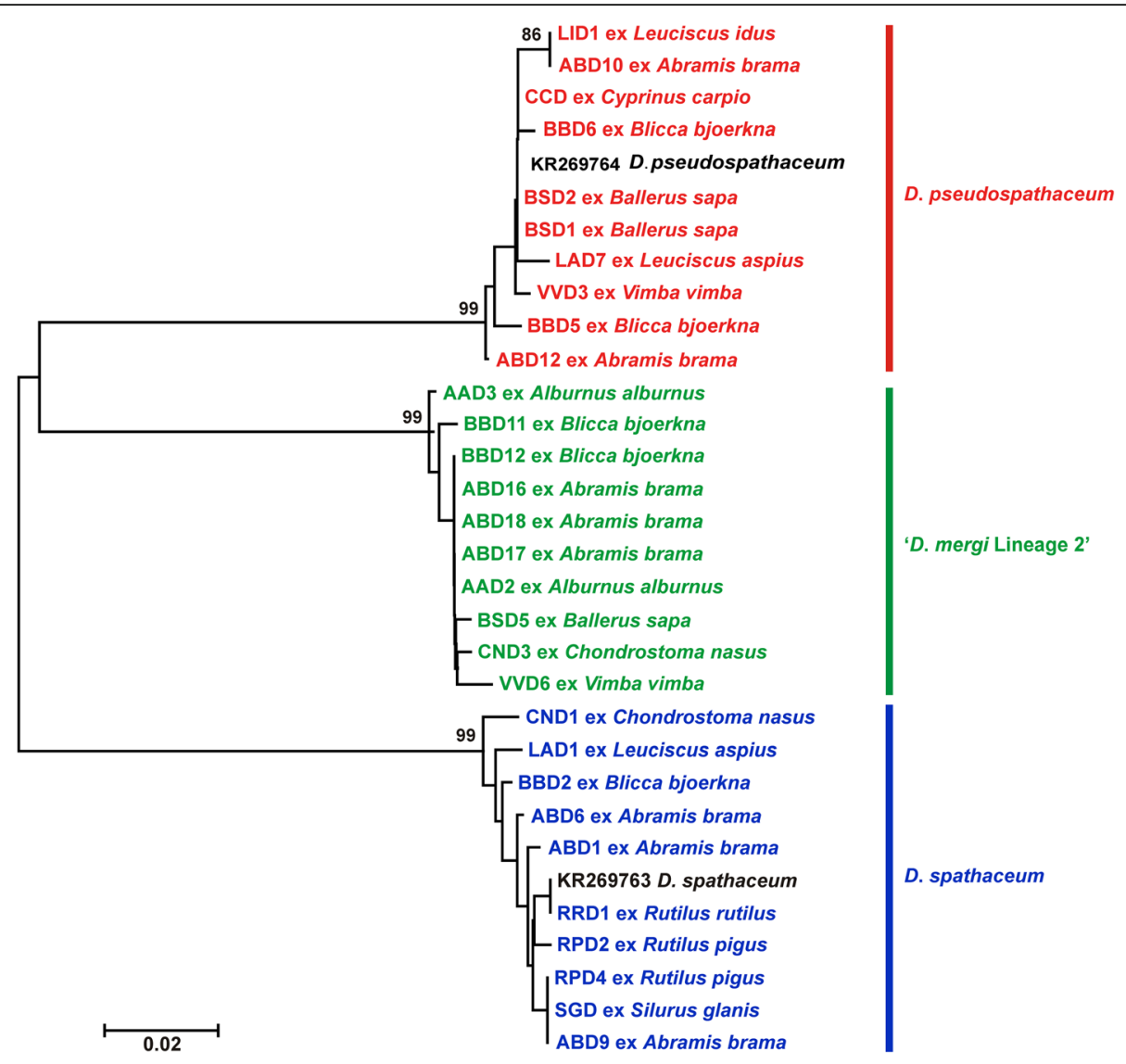

Fig. 5 Neighbour-joining (NJ) phylogram for Diplostomum spp. reconstructed using 30 newly generated and two nad3 sequences retrieved from GenBank. The scale-bar indicates the expected number of substitutions per site. Codes for the newly sequenced isolates are provided in Table 2

oval, $51-80 \times 46-69(62 \times 57)$. Pseudosuckers strongly muscular, elongate-oval, $58-90 \times 31-51(76 \times 41)$. Oral opening terminal; prepharynx absent; pharynx elongateoval, $32-47 \times 20-39(40 \times 28)$; oesophagus short, bifurcates close posterior to pharynx; caeca long, narrow, reach posterior to holdfast organ. Ventral sucker transversely oval, $34-64 \times 38-66(50 \times 56)$, smaller or equal to oral sucker (sucker width ratio 1:0.83-1.19 (1:1.01), posterior to mid-body length. Distance from anterior extremity of body to ventral sucker 191-365 (262). Holdfast organ relatively small, transversely oval, bipartite, contiguous with ventral sucker, 71-153 × 78-180 $(108 \times 124)$. Excretory vesicle small, V-shaped; reserve excretory system of diplostomid type; excretory

Table 4 Levels of divergence (p-distance in \%) for cox1 and nad3 gene sequences in interspecific comparisons of Diplostomum spp.

\begin{tabular}{lll}
\hline Species comparison & cox1 & nad3 \\
\hline D. pseudospathaceum vs D. spathaceum & $9.0-10.7$ & $15.7-17.4$ \\
D. spathaceum vs 'D. mergi Lineage 2' & $10.0-11.7$ & $15.4-16.8$ \\
D. pseudospathaceum vs 'D. mergi Lineage 2' & $11.2-12.9$ & $14.6-16.2$ \\
\hline
\end{tabular}

concretions relatively large, 171-346 (246) in number, grouped into 2 lateral extracaecal [106-254 (179) excretory concretions] and 1 median [39-109 (67) excretory concretions] fields. Hindbody 34-59 (44) long.

\section{Remarks}

The morphology of the present metacercariae of $D$. spathaceum (Fig. 6a) agrees with the descriptions of metacercariae of $D$. spathaceum by Faltýnková et al. [16] and Pérez-del-Olmo et al. [3] with some variations. The present live specimens differ from the live material described by Faltýnková et al. [16] by having on average shorter and wider body, somewhat larger pseudosuckers and ventral sucker, narrower holdfast organ and a different sucker width ratio (mean 1:1.01 vs 1:0.84) (also see Table 5). Similarly, the present fixed specimens differ from the fixed material described by Faltýnková et al. [16] and Pérez-del-Olmo et al. [3] in having on average shorter and wider body and larger pseudosuckers and ventral sucker and a distinctly wider holdfast organ. The number of excretory concretions in $D$. spathaceum falls within the range provided by Shigin [1] but the mean is distinctly higher: 171-346 (246) vs 117-401 (143). 
Table 5 Comparative metrical data for metacercariae of Diplostomum spathaceum

\begin{tabular}{|c|c|c|c|c|c|c|c|c|}
\hline \multirow{3}{*}{$\begin{array}{l}\text { Host } \\
\text { Source } \\
\text { Variable }\end{array}$} & \multirow{2}{*}{\multicolumn{2}{|c|}{$\begin{array}{l}\text { Multiple hosts }^{a} \\
\text { Present study } \\
\text { Fixed }\end{array}$}} & \multicolumn{4}{|c|}{$\begin{array}{l}\text { Gasterosteus aculeatus L.; Salvelinus alpinus (L.) } \\
\text { Faltýnková et al. [16] }\end{array}$} & \multirow{2}{*}{\multicolumn{2}{|c|}{$\begin{array}{l}\text { Cyprinus carpio L. } \\
\text { Pérez-del-Olmo et al. [3] } \\
\text { Fixed }\end{array}$}} \\
\hline & & & \multicolumn{2}{|l|}{ Live } & \multicolumn{2}{|l|}{ Fixed } & & \\
\hline & Range $(n=21)$ & Mean & Range & Mean & Range & Mean & Range & Mean \\
\hline$\overline{B L}$ & $288-415$ & 346 & $360-570$ & 498 & $262-574$ & 376 & $277-453$ & 376 \\
\hline BW & $241-333$ & 288 & $252-332$ & 286 & $171-313$ & 235 & 198-295 & 248 \\
\hline $\mathrm{HL}$ & 17 & 17 & $36-80$ & 53 & $22-67$ & 41 & $10-26$ & 16 \\
\hline PSL & $46-61$ & 53 & - & - & $35-40$ & 37 & $44-55$ & 48 \\
\hline PSW & $24-36$ & 29 & - & - & - & - & $22-30$ & 26 \\
\hline OSL & $40-54$ & 47 & $44-65$ & 57 & $44-64$ & 52 & $40-57$ & 45 \\
\hline OSW & $37-52$ & 46 & $44-72$ & 60 & $41-72$ & 50 & $36-41$ & 39 \\
\hline $\mathrm{PHL}$ & $30-42$ & 38 & $36-51$ & 42 & 29-45 & 35 & $29-43$ & 37 \\
\hline PHW & $16-26$ & 21 & $20-32$ & 26 & $16-19$ & 17 & $19-26$ & 23 \\
\hline VSL & $38-51$ & 45 & $35-55$ & 45 & $40-56$ & 49 & $30-43$ & 38 \\
\hline VSW & $48-61$ & 54 & $38-62$ & 50 & $34-53$ & 43 & $33-48$ & 43 \\
\hline AVS & $135-248$ & 181 & - & - & - & - & - & - \\
\hline $\mathrm{HOL}$ & $67-99$ & 84 & $78-131$ & 104 & $72-82$ & 77 & $63-89$ & 75 \\
\hline HOW & $92-130$ & 112 & 83-181 & 131 & 63-95 & 81 & 59-90 & 80 \\
\hline
\end{tabular}

Abbreviations: $B L$ body length, $B W$ body width, $H L$ hindbody length, PSL pseudosucker length, PSW pseudosucker width, OSL oral sucker length, OSW oral sucker width, $P H L$ pharynx length, $P H W$ pharynx width, VSL ventral sucker length, VSW ventral sucker width, AVS distance from anterior extremity of body to ventral sucker, $\mathrm{HOL}$ holdfast organ length, HOW holdfast organ width

${ }^{a}$ Acipenser ruthenus L.; Abramis brama (L.); Blicca bjoerkna (L.); Chondrostoma nasus (L.); Leuciscus aspius (L.); Rutilus pigus (Lacépède); Rutilus rutilus (L.); Vimba vimba (L.); Silurus glanis L.

Our study adds 8 fish species to the hosts of $D$. spathaceum in Europe confirmed by molecular evidence. Previous records include Gasterosteus aculeatus L. in Germany [7]; G. aculetaus and Salvelinus alpinus (L.) in Iceland [9]; Misgurnus anguillicaudatus (Cantor), S. glanis and P. willkommii in Spain [3]; and Perca fluviatilis L. in Italy and S. glanis in Romania [6]. Among these hosts, cyprinids predominate (7 species) and are more diverse; a very high prevalence $(75 \%)$ was also registered in a cyprinid (A. brama; present study).

\section{Diplostomum pseudospathaceum Niewiadomska, 1984}

Hosts: Abramis brama (L.), Ballerus sapa (Pallas), Blicca bjoerkna (L.), Cyprinus carpio L., Leuciscus aspius (L.), L. idus (L.), Vimba vimba (L.) (Teleostei: Cyprinidae); Lota lota (L.) (Teleostei: Lotidae), Gymnocephalus schraetser (L.) (Teleostei: Percidae).

Prevalence: A. brama: 50\% (20/40, S); B. sapa: $1 / 1$ (S); B. bjoerkna: $3 / 5(\mathrm{H}), 5 / 8(\mathrm{~S})$; C. carpio: $1 / 3(\mathrm{~S})$; L. aspius: 2/5 (H), 3/4 (S); L. idus: $1 / 1(\mathrm{~S}) ;$ V. vimba: $1 / 5(\mathrm{H}), 1 / 4$ (S); L. lota: $1 / 2(\mathrm{H}) ;$ G. schraetser: 1/5 (H).

Representative DNA sequences: KY653987-KY654016 (cox1); KY654047-KY654056 (nad3).

\section{Description}

[Based on 18 live metacercariae. Metrical data for fixed material are provided in Table 6; Fig. 6b.] Body elongate- oval, $325-490 \times 234-410(406 \times 306)$, with maximum width just anterior to ventral sucker. Oral sucker elongateoval, $48-65 \times 43-58(55 \times 50)$. Pseudosuckers strongly muscular, elongate-oval, $42-73 \times 26-43(54 \times 33)$. Oral opening terminal; prepharynx short or absent; pharynx elongate-oval, $31-52 \times 19-37(38 \times 24)$; oesophagus short, bifurcates close posterior to pharynx; caeca long, narrow, reach posterior to holdfast organ. Ventral sucker transversely oval, $37-56 \times 45-66(47 \times 55)$, smaller or larger than oral sucker [sucker width ratio 1:0.93-1.35 (1:1.11)], slightly posterior to mid-body length. Distance from anterior extremity of body to ventral sucker 177-279 (216). Holdfast organ relatively small, transversely oval, bipartite, contiguous with ventral sucker, 69-111 × 88$170(90 \times 115)$. Excretory vesicle small, V-shaped; reserve excretory system of diplostomid type; excretory concretions small, 185-360 (241) in number, grouped into 2 lateral extracaecal [122-244 (164) excretory concretions] and 1 median [57-116 (77) excretory concretions] fields. Hindbody 19-47 (31) long.

\section{Remarks}

The present metacercariae were identified as D. pseudospathaceum based on molecular data. The metrical data for the present material (fixed specimens) exhibit overlapping ranges with the data for experimentally developed metacercariae of $D$. pseudospathaceum described by Niewiadomska [26] but differ in the possesion of on 
Table 6 Comparative metrical data for metacercariae of Diplostomum spp.

\begin{tabular}{|c|c|c|c|c|c|c|c|c|c|}
\hline $\begin{array}{l}\text { Species } \\
\text { Host }\end{array}$ & $\begin{array}{l}\text { Diplostomum } \\
\text { pseudospathace } \\
\text { Multiple hosts }^{\mathrm{a}}\end{array}$ & & $\begin{array}{l}\text { Diploston } \\
\text { pseudosp } \\
\text { Cyprinus }\end{array}$ & $\begin{array}{l}m \\
\text { haceum } \\
\text { rpio L. }\end{array}$ & $\begin{array}{l}\text { 'Diplostomum m } \\
\text { Lineage } 2 \text { ' } \\
\text { Multiple hosts }\end{array}$ & & $\begin{array}{l}\text { Diplostomum sp. A } \\
\text { Blicca bjoerkna (L.) }\end{array}$ & $\begin{array}{l}\text { Diplostomum sp. B } \\
\text { Carassius gibelio (Bloch) }\end{array}$ & $\begin{array}{l}\text { Diplostomum sp. C } \\
\text { Rutilus rutilus (L.) }\end{array}$ \\
\hline \multirow[t]{2}{*}{ Source } & \multicolumn{2}{|l|}{ Present study } & \multicolumn{2}{|c|}{ Niewiadomska [26] } & \multicolumn{2}{|l|}{ Present study } & \multirow{2}{*}{$\begin{array}{l}\text { Present study } \\
\text { Fixed }\end{array}$} & \multirow{2}{*}{$\begin{array}{l}\text { Present study } \\
\text { Fixed }\end{array}$} & \multirow{2}{*}{$\begin{array}{l}\text { Present study } \\
\text { Fixed }\end{array}$} \\
\hline & Fixed & & Fixed & & Fixed & & & & \\
\hline Variable & Range $(n=24)$ & Mean & Range & Mean & Range $(n=18)$ & Mean & $n=1$ & $n=1$ & $n=1$ \\
\hline $\mathrm{BL}$ & $288-447$ & 364 & $347-458$ & 381 & $362-485$ & 420 & 338 & 426 & 381 \\
\hline BW & 234-301 & 264 & $162-296$ & 201 & $242-338$ & 287 & 242 & 304 & 278 \\
\hline $\mathrm{HL}$ & $19-19$ & 19 & - & - & $14-45$ & 26 & 20 & 19 & 16 \\
\hline PSL & $40-65$ & 52 & - & - & $52-68$ & 60 & $47-52$ & $56-58$ & $61-67$ \\
\hline PSW & $25-35$ & 30 & - & - & $31-36$ & 34 & - & - & - \\
\hline OSL & $39-56$ & 47 & $42-52$ & 45.8 & $41-53$ & 47 & 37 & 46 & 47 \\
\hline OSW & $36-53$ & 44 & $30-51$ & 37.7 & $34-49$ & 43 & 44 & 41 & 47 \\
\hline PHL & $32-45$ & 38 & $28-35$ & 31.8 & $30-45$ & 38 & 30 & 41 & 30 \\
\hline PHW & $19-25$ & 21 & $17-30$ & 20.4 & $19-23$ & 22 & 20 & 22 & - \\
\hline VSL & $33-53$ & 42 & $34-42$ & 38.9 & $40-62$ & 51 & 51 & 51 & 43 \\
\hline VSW & $43-56$ & 51 & $35-51$ & 42.2 & $49-70$ & 61 & 64 & 59 & 49 \\
\hline AVS & $158-243$ & 191 & - & - & $174-261$ & 208 & 143 & 215 & 174 \\
\hline $\mathrm{HOL}$ & $68-96$ & 82 & $62-81$ & 67.5 & $95-115$ & 104 & 65 & 115 & - \\
\hline HOW & 79-126 & 99 & $54-76$ & 61.7 & 102-187 & 136 & 106 & 136 & - \\
\hline
\end{tabular}

Abbreviations: $B L$ body length, $B W$ body width, $H L$ hindbody length, $P S L$ pseudosucker length, $P S W$ pseudosucker width, OSL oral sucker length, OSW oral sucker width, $P H L$ pharynx length, PHW pharynx width, VSL ventral sucker length, VSW ventral sucker width, AVS distance from anterior extremity of body to ventral sucker, HOL holdfast organ length, HOW holdfast organ width

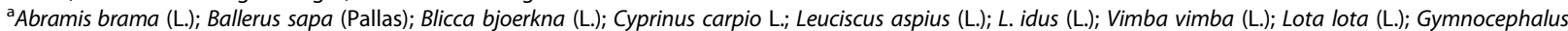
schraetser (L.)

${ }^{\mathrm{b}}$ Abramis brama (L.); Alburnus alburnus (L.); Ballerus sapa (Pallas); Blicca bjoerkna (L.); Chondrostoma nasus (L.); Vimba vimba (L.)

average shorter and wider body, wider suckers and distinctly wider holdfast organ (Table 6). Shigin [1] reported 151-309 (234) excretory concretions for D. pseudospathaceum (as $D$. chromatophorum); these values agree very well with our observations, i.e. 185-360 (241).

Our study reports nine fish hosts for $D$. pseudospathaceum in Europe confrmed by sequencing. Previous molecularly identified records in fishes are few: G. aculeatus in Germany [7] and C. carpio and S. glanis in Romania [6]. Among the hosts studied here, cyprinids predominated (7 species) with a high prevalence in $A$. brama (50\%).

'Diplostomum mergi Lineage 2' sensu Georgieva et al. (2013)

Hosts: Abramis brama (L.), Alburnus alburnus (L.), Ballerus sapa (Pallas), Blicca bjoerkna (L.), Chondrostoma nasus (L.), Vimba vimba (L.) (Teleostei: Cyprinidae).

Prevalence: A. brama: 58\% (23/40, S); A. alburnus: $3 / 5$ (H), 1/3 (S); B. sapa: 1/8 (H), 1/1 (S); B. bjoerkna: $1 / 5$ (H), 2/8 (S); C. nasus: $1 / 4$ (S); V. vimba: $4 / 5$ (H).

Representative DNA sequences: KY654017-KY654033 (cox1); KY654057-KY654066 (nad3).

\section{Description}

[Based on 8 live metacercariae. Metrical data for fixed material are provided in Table 6; Fig. 6c.] Body elongate-oval, $456-529 \times 256-382(490 \times 328)$, with maximum width just anterior to ventral sucker. Oral sucker subspherical, $48-57 \times 46-61(52 \times 53)$. Pseudosuckers elongate-oval, $69-73 \times 32-40(67 \times 36)$. Oral opening terminal; prepharynx short; pharynx elongate-oval, $29-40 \times 23-34(35 \times 26)$; oesophagus short, bifurcates close posterior to pharynx; caeca long, narrow, reach posterior to holdfast organ. Ventral sucker transversely oval, 54-61 $\times$ 64-71 $(57 \times 67)$, distinctly larger than oral sucker (sucker width ratio 1:1.14-1.31 (1:1.25), at mid-body length. Distance from anterior extremity of body to ventral sucker 205-265 (237). Holdfast organ large, transversely oval, bipartite, contiguous with ventral sucker, $120-158 \times 152-205(134 \times 174)$. Excretory vesicle small, V-shaped; reserve excretory system of diplostomid type; excretory concretions predominantly medium-sized, 316-440 (372) in number, grouped into 2 lateral extracaecal [229-360 (285) excretory concretions] and 1 median [58-122 (87) excretory concretions] fields. 

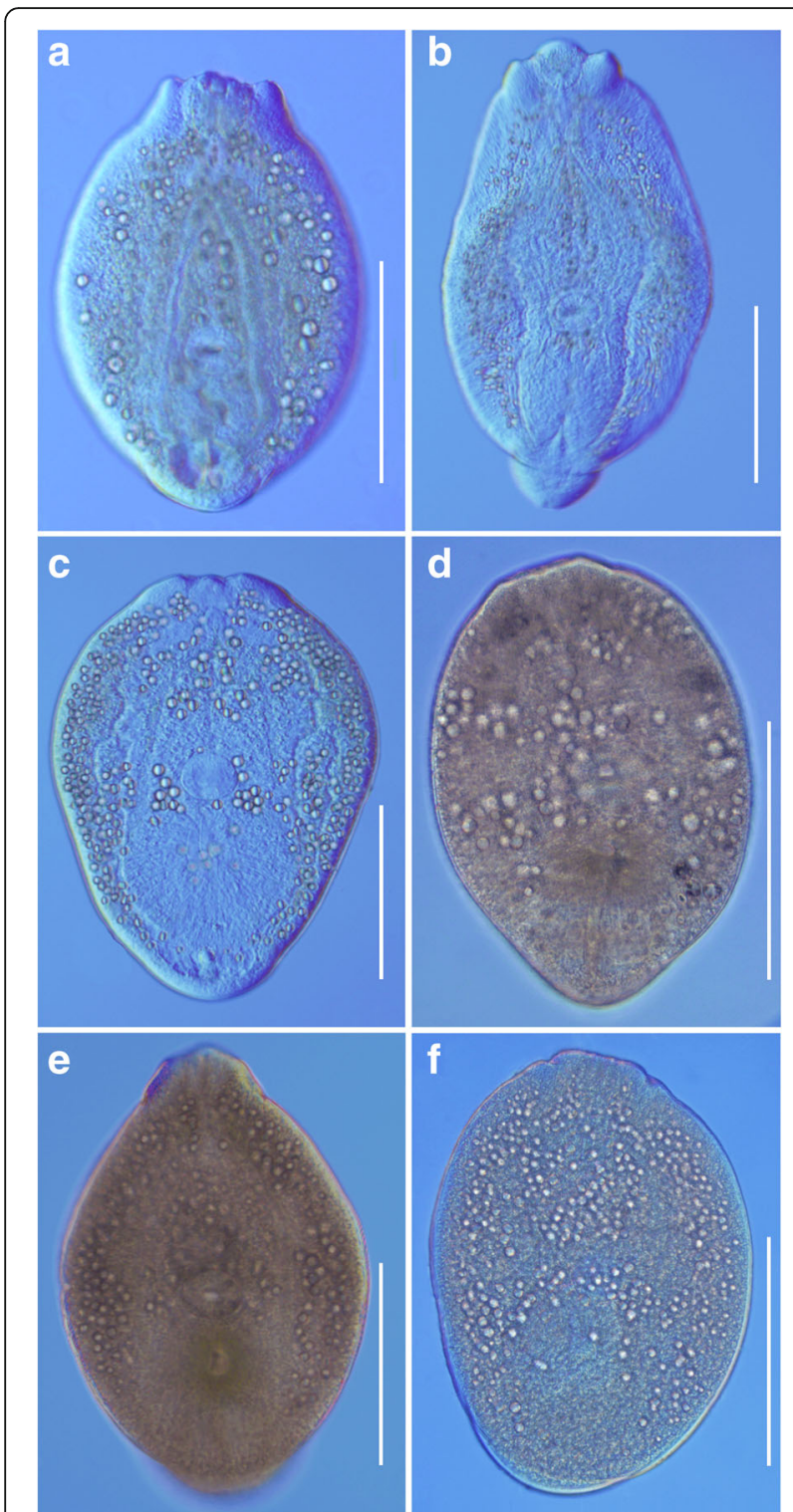

Fig. 6 Metacercariae of Diplostomum spp. (a-c, live; d-f, fixed). a $D$. spathaceum from the eye lens of Rutilus pigus (hologenophore; GenBank KY653979 and KY654043). b D. pseudospathaceum from the eye lens of Abramis brama (hologenophore; GenBank KY653989 and KY654048). c 'D. mergi Lineage 2 ' from the eye lens of Abramis brama (hologenophore; GenBank KY654020 and KY654059). d Diplostomum $\mathrm{sp}$. A from the eye lens of Blicca bjoerkna (hologenophore; GenBank KY654034). e Diplostomum sp. B from the eye lens of Carassius gibelio (hologenophore; GenBank KY654035). f Diplostomum sp. C from the eye lens of Rutilus rutilus (hologenophore; GenBank KY654036). Scale-bars: $200 \mu \mathrm{m}$

\section{Remarks}

Shigin [1] suggested that the large size and number [702-854 (772)] of the excretory concretions in the metacercariae of $D$. mergi (sensu lato) clearly distinguish this species from all lens-infecting forms. However, molecular analyses by Georgieva et al. [7] and Selbach et al. [10] revealed the presence of at least four cryptic species within this complex. The present material is characterised by a distinctly smaller number of excretory concretions, i.e. 316-443 (372) thus adding morphological evidence to the genetic differentiation of ' $D$. mergi Lineage 2'.

To date, 'D. mergi Lineage 2' has only been recorded/ sequenced in Europe from snails in Germany: three cercarial isolates from $R$. auricularia from Hengsteysee [7] and 13 cercarial isolates from the same host in Baldeneysee, Hengsteysee and Sorpetalsperre [10]. Our study, therefore partially elucidates the life-cycle of this species, providing the first data for the second intermediate hosts in Europe comprising six new host records, all cyprinids. Similarly to the other two Diplostomum spp. reported here, high prevalence of infection (58\%) was detected in A. brama. It is worth noting that a single metacercarial isolate has been sequenced from A. brama in China [6].

\section{Diplostomum sp. A}

Host: Blicca bjoerkna (L.) (Teleostei: Cyprinidae).

Prevalence: 1/8 (Slovakia).

Representative DNA sequence: KY654034 (cox1).

\section{Description}

[Based on 1 fixed metacercaria; see also Table 6, Fig. 6d.] Body elongate-oval, $338 \times 242$, with maximum width at level of ventral sucker. Oral sucker transversely oval, $37 \times 44$. Pseudosuckers distinct, muscular, 47-52 long. Oral opening terminal; prepharynx absent; pharynx elongate-oval, $30 \times 20$; oesophagus short. Ventral sucker transversely oval, $51 \times 64$, larger than oral sucker (sucker width ratio 1:1.45), located at mid-body length. Distance from anterior extremity of body to ventral sucker 143 . Holdfast organ small, transversely oval, bipartite, contiguous with ventral sucker, $65 \times 106$. Excretory vesicle small, V-shaped; reserve excretory system of diplostomid type; excretory concretions very large, 154 in number, grouped into 2 lateral extracaecal (107 excretory concretions) and 1 median (47 excretory concretions) fields. Hindbody 20 long.

\section{Diplostomum sp. B}

Host: Carassius gibelio (Bloch) (Teleostei: Cyprinidae). Prevalence: 1/6 (Slovakia).

Representative DNA sequence: KY654035 (cox1).

\section{Description}

[Based on 1 fixed metacercaria; see also Table 6, Fig. 6e.] Body elongate-oval, $426 \times 304$, with maximum width at level of ventral sucker. Oral sucker elongate-oval, 
$46 \times$ 41. Pseudosuckers muscular, 56-58 long. Oral opening terminal; prepharynx short; pharynx elongateoval, $41 \times 22$; oesophagus short, bifurcates close posterior to pharynx; caeca long, narrow, reach posterior to holdfast organ. Ventral sucker transversely oval, $51 \times 59$, larger than oral sucker (sucker width ratio 1:1.44), located at mid-body length. Distance from anterior extremity of body to ventral sucker 215. Holdfast organ large, transversely oval, bipartite, contiguous with ventral sucker, $115 \times 136$. Excretory vesicle small, V-shaped; reserve excretory system of diplostomid type; excretory concretions predominantly large, 261 in number, grouped into 2 lateral extracaecal (168 excretory concretions) and 1 median (93 excretory concretions) fields. Hindbody 19 long.

\section{Diplostomum sp. C}

Host: Rutilus rutilus (L.) (Teleostei: Cyprinidae).

Prevalence: 1/8 (Slovakia).

Representative DNA sequence: KY654036 (cox1).

\section{Description}

[Based on 1 fixed metacercariae. Metrical data for the isolate are provided in Table 6; Fig. 6f.] Body oval, $381 \times 278$, with maximum width at level of ventral sucker. Oral sucker spherical, $47 \times 47$. Pseudosuckers strongly muscular, 61-67 long. Oral opening terminal; prepharynx short; pharynx 30 long. Ventral sucker transversely oval, $43 \times 49$, similar in size to oral sucker (sucker width ratio 1:1.04), located at mid-body length. Distance from anterior extremity of body to ventral sucker 174. Holdfast organ transversely oval, bipartite, contiguous with ventral sucker. Excretory vesicle small, V-shaped; reserve excretory system of diplostomid type; excretory concretions predominantly small, 482 in number, grouped into 2 lateral extracaecal (334 excretory concretions) and 1 median (148 excretory concretions) fields. Hindbody 16 long.

\section{Discussion}

Parasite diversity in fishes from the River Danube has been studied extensively in the past (see Moravec [27]). However, remarkably little is known about the actual species diversity of the metacercariae of the genus Diplostomum. These have been typically reported as $D$. spathaceum, without any morphological evidence confirming species identification, or left unidentified (see Moravec [27] for details of the records). Due to the failure in achieving species identification of the metacercariae based on morphology, this practice is observed in a number of recent ecological studies of fish parasites from the River Danube (e.g. [28-32]). Recently, a single cox 1 sequence for $D$. pseudospathaceum has been published from S. glanis in the River Danube in Romania [6].

The present study is the first taxonomically broad screening of fish hosts to provide data on the diversity of Diplostomum spp. from the River Danube applying molecular identification methods. The analyses based on the newly generated and published $\operatorname{cox} 1$ sequences demonstrated the presence of three species/species-level genetic lineages of Diplostomum, i.e. D. spathaceum, $D$. pseudospathaceum and 'D. mergi Lineage 2', and three single isolates potentially representing distinct species, i.e. Diplostomum spp. A-C. Our approach ensured a refined taxonomic resolution and allowed an assessment of the host ranges of the three most frequent Diplostomum spp. and to partly elucidate the life-cycle of one species. The large number of isolates from a wide range of hosts examined led to the detection of the somewhat higher level of mean intraspecific divergence for $D$. spathaceum and ' $D$. mergi Lineage 2' compared with previous data: 0.82 vs $0.43 \%$ [7] and $0.53 \%$ [10], and $0.47 v s$ $0 \%$ [7] and $0.30 \%$ [10], respectively.

Our novel data for host ranges of $D$. spathaceum, $D$. pseudospathaceum and ' $D$. mergi Lineage 2', based on molecular identification of the metacercariae, indicate that the transmission of these species in the River Danube is primarily associated with cyprinid fishes as second intermediate hosts. Twelve out of fourteen cyprinid species were infected with at least one species of Diplostomum; the largest number of species/lineages (4 out of 6) was detected in B. bjoerkna. Diplostomum spathaceum was also found in A. ruthenus (Acipenseridae) and $S$. glanis (Siluridae) and D. pseudospathaceum was recovered in G. schraetser (Percidae) and Lota lota (Lotidae). All three species of Diplostomum exhibited remarkably high prevalence in A. brama, the most well-sampled species. Although the lack of infections with Diplostomum spp. in 12 out of the 28 species of fish examined may be due to the small sample sizes, infections were detected in a large number of similarly under-sampled species, i.e. the acipenserid $A$. ruthenus (D. spathaceum), the cyprinids $A$. alburnus (' $D$. mergi Lineage 2 '), B. sapa (D. pseudospathaceum and ' $D$. mergi Lineage 2'), C. gibelio (Diplostomum sp. B), C. nasus (D. spathaceum and ' $D$. mergi Lineage 2'), C. carpio (D. pseudospathaceum), $L$. aspius (D. spathaceum and D. pseudospathaceum), L. idus (D. pseudospathaceum), R. pigus (D. spathaceum), R. rutilus (D. spathaceum and Diplostomum sp. C), V. vimba (D. spathaceum, $D$. pseudospathaceum and ' $D$. mergi Lineage 2'), the lotid L. lota (D. pseudospathaceum), the percid G. schraetser (D. pseudospathaceum) and the silurid S. glanis (D. spathaceum). These data indicate that the species/lineages reported here may parasitise a wide range of hosts. The lack of specific host-related pattern of genetic structuring, illustrated by the haplotype networks for $D$. 
spathaceum and D. pseudospathaceum, based on the novel data and the pattern of shared haplotypes with isolates from fish hosts of the Cobitidae, Gasterosteidae, Percidae, Salmonidae and Siluridae (detailed in Table 3), also tend to support this suggestion. Furthermore, the apparent lack of host-specificity for $D$. spathaceum and $D$. pseudospathaceum is confirmed by the wide host ranges (17 fish species of 7 families and 12 host species of 5 families, respectively) in the expanded datasets comprising the cox 1 sequences available to date (Figs. 3b, 4b; Additional file 2: Table S2). The most common haplotypes exhibited low host-specificity at the level of both host species (our novel data) and host family (expanded datasets).

Regarding the geographical distribution, the present comparisons with all published sequences revealed haplotypes with a wide Palaearctic distribution for two of the species, reported from Iraq and China by Locke et al. [6], i.e. D. spathaceum (H2: Iraq, China; H5, H7 and H10: Iraq; H13: China); 'D. mergi Lineage 2' (H7: China); a number of haplotypes of $D$. spathaceum $(n=30)$ are currently known from Asia only (see Locke et al. [6]; Additional file 2: Table S2).

Our study represents the first record of ' $D$. mergi Lineage 2' in a fish host in Europe and is the first to provide a morphological description of the metacercaria. The new isolates clustered together, and exhibited additional shared haplotypes, with cercarial isolates sequenced by Georgieva et al. [7] and Selbach et al. [10]. Thus, the life-cycle of this lineage was partially elucidated using molecular data, with the pulmonate snail $R$. auricularia acting as the first intermediate host and six cyprinid fishes (A. alburnus, A. brama, B. bjoerkna, B. sapa, $C$. nasus and $V$. vimba) acting as second intermediate hosts. The cercaria of ' $D$. mergi Lineage 2' was described in detail by Selbach et al. [10] who differentiated it from the cercaria of $D$. mergi sensu Niewiadomska \& Kiselienė, 1994 [33] by having furcae longer than the tail stem and by morphometry, and from the cercariae of the four species within the " $D$. mergi" species complex by five unique morphometric features (see Selbach et al. [10] for details). The present metacercariae exhibited markedly smaller number of excretory concretions in comparison with the metacercariae of $D$. mergi (sensu lato) (mean 372 vs 772; see [1]) and showed morphometric differences from the metacercariae of the other lens-infecting species, D. spathaceum and D. pseudospathaceum. These data, in association with the genetic evidence, support the distinct species status of ' $D$. mergi Lineage 2'; however, formal description of the species would require the discovery of the adult stage. The distribution of this species-level genetic lineage is apparently wider, and not restricted to Europe, since Locke et al. [6] reported a single sequence from a metacercaria in the cyprinid A. brama from China. Further studies would add to our knowledge of haplotype diversity, host ranges and geographical distribution of this lineage.

Brabec et al. [25] characterised the complete mitochondrial genomes of the two closely related species, D. spathaceum and D. pseudospathaceum and carried out a comparative genome assessment. These authors revealed that the cox 1 gene and its 'barcode' region, currently applied for molecular identification, represent the most conserved protein-coding regions of the mitochondrial genome of Diplostomum spp. and identified nad4 and nad5 genes as most promising molecular diagnostic markers. In the pilot nad gene sequencing carried out here, we opted for nad3 gene, a slightly more conserved in comparison to the nad4 and nad5 genes, because the identification based on cox 1 revealed the presence of a lineage of the " $D$. mergi" species complex that was shown to be rather distant to the two sibling species studied by Brabec et al. [25] (e.g. [7, 10]). Our results indicate that the newly designed primers can be used for successful amplification of nad 3 within the " $D$. mergi" complex and possibly in other distantly related lineages of Diplostomum; the markedly higher levels of interspecific divergence compared to cox 1 indicate that the nad3 gene is a good candidate marker for multi-gene approaches to systematic estimates within the genus.

\section{Conclusions}

The first exploration of the species diversity and host ranges of Diplostomum spp., based on molecular and morphological evidence from a broad range of fish hosts in the River Danube (Hungary and Slovakia), revealed the presence of three species/species-level genetic lineages of Diplostomum, i.e. D. spathaceum, D. pseudospathaceum and 'D. mergi Lineage 2', and three single isolates potentially representing distinct species. The most frequently found Diplostomum spp. exhibited a low host-specificity, predominantly infecting a wide range of cyprinid fishes but also species of distant fish families such as the Acipenseridae, Lotidae, Percidae and Siluridae. Our study provided the first $\operatorname{cox} 1$ and nad 3 sequences associated with a morphological characterisation for metacercariae of ' $D$. mergi Lineage 2 ' in a fish host in Europe and partially elucidated the life-cycle of this species using molecular data. The novel sequence data will advance further ecological studies on the distribution and host ranges of these important fish parasites in Europe.

\section{Additional files}

Additional file 1: Table S1. Summary data for the sequences from isolates of Diplostomum spp. isolates retrieved from the GenBank database and used in the phylogenetic analyses. (DOC $67 \mathrm{~kb}$ ) 
Additional file 2: Table S2. Summary data for the sequences for Diplostomum spathaceum and D. pseudospathaceum from metacercarial isolates used in the expanded haplotype networks. (DOCX $31 \mathrm{~kb}$ )

\section{Acknowledgements}

We are grateful to Jan Brabec and Roman Kuchta (Institute of Parasitology, Biology Centre of the Czech Academy of Sciences) and Tibor Eros (Balaton Limnological Institute, Hungarian Academy of Sciences) for their invaluable help during material collection. We thank the three anonymous reviewers for their constructive comments and suggestions.

\section{Funding}

This research was partially supported by the Czech Science Foundation, grants 15-14198S (SG and AK) and ECIP P505/12/G112 (OK); the Research \& Development Operational Programme funded by the ERDF (code ITMS: 26220120022) (0.3) (MO). SG benefited from a postdoctoral fellowship of the Czech Academy of Sciences. This is contribution number 214 from the NWUWater Research Group.

\section{Availability of data and materials}

The data supporting the conclusions of this article are included within the article and its additional files. The newly generated sequences are submitted to the GenBank database under the accession numbers KY653961-KY654066.

\section{Authors' contributions}

SG and MO: obtained the samples, undertook the identification and morphological characterisation of the isolates. OK and SG: carried out the morphological analysis, sequencing, performed the phylogenetic analyses and drafted the MS. AK: conceived and coordinated the study, discussed the results and helped draft the manuscript. All authors read and approved the final manuscript.

\section{Ethics approval}

All applicable institutional, national and international guidelines for the care and use of animals were followed.

\section{Consent for publication}

Not applicable.

\section{Competing interests}

The authors declare that they have no competing interests.

\section{Publisher's Note}

Springer Nature remains neutral with regard to jurisdictional claims in published maps and institutional affiliations.

\section{Author details \\ ${ }^{1}$ Water Research Group, Unit for Environmental Sciences and Management, Potchefstroom Campus, North-West University, Potchefstroom 2520, South Africa. ${ }^{2}$ Institute of Parasitology, Biology Centre of the Czech Academy of Sciences, Branišovská 31, 37005 České Budějovice, Czech Republic. ${ }^{3}$ Institute of Ecology, Nature Research Centre, Akademijos 2, 08412 Vilnius, Lithuania. ${ }^{4}$ Institute of Parasitology, Slovak Academy of Sciences, Hlinkova 3, 04001 Košice, Slovak Republic.}

Received: 25 May 2017 Accepted: 1 November 2017 Published online: 02 December 2017

\section{References}

1. Shigin AA. [Trematode fauna of the USSR. Genus Diplostomum. Metacercariae.] Moscow: Nauka; 1986 (In Russian).

2. Chappell LH, Hardie $\amalg$, Secombes CJ. Diplostomiasis: the disease and hostparasite interactions. In: Pike AW, Lewis JW, editors. Parasitic diseases of fish. Tresaith, Dyfed, UK: Samara Publishing Ltd.; 1994. p. 59-86.

3. Pérez-del-Olmo A, Georgieva S, Pula HJ, Kostadinova A. Molecular and morphological evidence for three species of Diplostomum (Digenea: Diplostomidae), parasites of fishes and fish-eating birds in Spain. Parasit Vectors. 2014;7:502.

4. Moszczynska A, Locke SA, McLaughlin JD, Marcogliese DJ, Crease TJ. Development of primers for the mitochondrial cytochrome $c$ oxidase I gene in digenetic trematodes illustrates the challenge of barcoding parasitic helminths. Mol Ecol Resour. 2009:9:75-82.

5. Locke SA, McLaughlin JD, Dayanandan S, Marcogliese DJ. Diversity, specificity and evidence of hybridization in Diplostomum spp. metacercariae in freshwater fishes is revealed by DNA barcodes and ITS sequences. Int J Parasitol. 2010a:40:333-43.

6. Locke SA, Al-Nasiri FS, Caffara M, Drago F, Kalbe M, Lapierre AR, et al. Diversity, specificity and speciation in larval Diplostomidae (Platyhelminthes: Digenea) in the eyes of freshwater fish, as revealed by DNA barcodes. Int J Parasitol. 2015:45:841-55.

7. Georgieva S, Soldánová M, Pérez-del-Olmo A, Dangel RD, Sitko J, Sures B, et al. Molecular prospecting for European Diplostomum (Digenea: Diplostomidae) reveals cryptic diversity. Int J Parasitol. 2013;43:57-72.

8. Behrmann-Godel J. Parasite identification, succession and infection pathways in perch fry (Perca fluviatilis): new insights through a combined morphological and genetic approach. Parasitology. 2013;140:509-20.

9. Blasco-Costa I, Faltýnková A, Georgieva S, Skírnisson K, Scholz T, Kostadinova A. Fish pathogens near the Arctic circle: molecular, morphological and ecological evidence for unexpected diversity of Diplostomum (Digenea: Diplostomidae) in Iceland. Int J Parasitol. 2014;44:703-15.

10. Selbach C, Soldánová M, Georgieva S, Kostadinova A, Sures B. Integrative taxonomic approach to the cryptic diversity of Diplostomum spp. in lymnaeid snails from Europe with a focus on the 'Diplostomum mergi' species complex. Parasit Vectors. 2015;8:300.

11. Kuhn JA, Kristoffersen R, Knudsen R, Jakobsen J, Marcogliese DJ, Locke SA, et al. Parasite communities of two three-spined stickleback populations in subarctic Norway - effects of a small spatial-scale host introduction. Parasitol Res. 2015:114:1327-39.

12. Soldánová M, Georgieva S, Roháčová J, Knudsen R, Kuhn JA, Henriksen EH et al. Molecular analyses reveal high species diversity of trematodes in a sub-Arctic lake. Int J Parasitol. 2017;47:327-45.

13. Locke SA, McLaughlin JD, Marcogliese DJ. Predicting the similarity of parasite communities in freshwater fishes using the phylogeny, ecology and proximity of hosts. Oikos. 2013;122:73-83.

14. Désilets HD, Locke SA, McLaughlin JD, Marcogliese DJ. Community structure of Diplostomum spp. (Digenea: Diplostomidae) in eyes of fish: main determinants and potential interspecific interactions. Int J Parasitol. 2013:43:929-39.

15. Galazzo DE, Dayanandan S, Marcogliese DJ, McLaughlin JD. Molecular systematics of some north American species of Diplostomum (Digenea) based on rDNA-sequence data and comparisons with European congeners. Can J Zool. 2002:80:2207-17.

16. Faltýnková A, Georgieva S, Kostadinova A, Blasco-Costa I, Scholz T, Skírnisson K. Diplostomum von Nordmann, 1832 (Digenea: Diplostomidae) in the subArctic: descriptions of the larval stages of six species discovered recently in Iceland. Syst Parasitol. 2014;89:195-213.

17. Tamura K, Stecher G, Peterson D, Filipski A, Kumar S. MEGA6: Molecular Evolutionary Genetics Analysisversion 6.0. Mol Biol Evol. 2013;30:2725-9.

18. Rozas J, Sanchez-DelBarrio JC, Messeguer X, Rozas R. DnaSP, DNA polymorphism analyses by thecoalescent and other methods. Bioinformatics. 2003:19:2496-7.

19. Clement M, Posada D, Crandall KA. TCS: a computer program to estimate gene genealogies. Mol Ecol. 2000:9:1657-9.

20. Templeton AR, Crandall KA, Sing CFA. Cladistic analysis of phenotypic associations with haplotypes inferred from restriction endonuclease mapping and DNA sequence data. III. Cladogram estimation. Genetics. 1992;132:619-33.

21. Telford MJ, Herniou EA, Russell RB, Littlewood DTJ. Changes in mitochondrial genetic codes as phylogenetic characters: two examples from the flatworms. Proc Natl Acad Sci USA. 2000;97:11359-64.

22. Ronquist F, Teslenko M, van der Mark P, Ayres DL, Darling A, Hohna S, et al. MrBayes 3.2: efficient Bayesian phylogenetic inference and model choice across a large model space. Syst Biol. 2012;61:539-42.

23. Darriba D, Taboada GL, Doallo R, Posada D. jModelTest 2: more models, new heuristics and parallel computing. Nat Methods. 2012;9:772.

24. Shigin AA. [Trematodes of the fauna of Russia and neighbouring regions. Genus Diplostomum. Adults]. Moscow: Nauka; 1993 (In Russian).

25. Brabec J, Kostadinova A, Scholz T, Littlewood DT. Complete mitochondrial genomes and nuclear ribosomal RNA operons of two species of Diplostomum (Platyhelminthes: Trematoda): a molecular resource for taxonomy and molecular epidemiology of important fish pathogens. Parasit Vectors. 2015;8:336 
26. Niewiadomska K. Verification of the life-cycles of Diplostomum spathaceum (Rudolphi, 1819) and D. pseudospathaceum Niewiadomska, 1984 (Trematoda, Diplostomidae). Syst Parasitol. 1986;8:23-31.

27. Moravec F. Checklist of the metazoan parasites of fishes of the Czech Republic and the Slovak Republic (1873-2000). Prague: Academia; 2001.

28. Ondračková $M$, Dávidová $M$, Pečínková $M$, Blažek $R$, Gelnar $M$, Valová Z, et al. Metazoan parasites of Neogobius fishes in the Slovak section of the river Danube. J Appl Ichthyol. 2005;21:345-9.

29. Ondračková M, Slováčková I, Trichkova T, Polačik M, Jurajda P. Shoreline distribution and parasite infection of black-striped pipefish Syngnathus abaster Risso, 1827 in the lower river Danube. J Appl Ichthyol. 2012;28:590-6.

30. Müehlegger JM, Jirsa F, Konecny R, Frank C. Parasites of Apollonia melanostoma (Pallas 1814) and Neogobius kessleri (Guenther 1861) (Osteichthyes, Gobiidae) from the Danube River in Austria. J Helminthol. 2010:84:87-92.

31. Jirsa F, Konecny R, Frank C, Sures B. The parasite community of the nase Chondrostoma nasus (L. 1758) from Austrian rivers. J Helminthol. 2011;85: 255-62.

32. Francová $K$, Ondračková $M$, Polačik $M$, Jurajda P. Parasite fauna of native and non-native populations of Neogobius melanostomus (Pallas, 1814) (Gobiidae) in the longitudinal profile of the Danube River. J Appl Ichthyol. 2011;27: 879-86.

33. Niewiadomska K, Kiselienè V. Diplostomum cercariae (Digenea) in snails from Lithuania. II. Survey of species. Acta Zool Lituan. 1994;39:179-86.

\section{Submit your next manuscript to BioMed Central and we will help you at every step:}

- We accept pre-submission inquiries

- Our selector tool helps you to find the most relevant journal

- We provide round the clock customer support

- Convenient online submission

- Thorough peer review

- Inclusion in PubMed and all major indexing services

- Maximum visibility for your research

Submit your manuscript at www.biomedcentral.com/submit 